\title{
Modelling collective decision making in groups and crowds: Integrating social contagion and interacting emotions, beliefs and intentions
}

\author{
Tibor Bosse • Mark Hoogendoorn • \\ Michel C. A. Klein - Jan Treur • C. Natalie van der Wal • \\ Arlette van Wissen
}

Published online: 21 June 2012

(C) The Author(s) 2012. This article is published with open access at Springerlink.com

\begin{abstract}
Collective decision making involves on the one hand individual mental states such as beliefs, emotions and intentions, and on the other hand interaction with others with possibly different mental states. Achieving a satisfactory common group decision on which all agree requires that such mental states are adapted to each other by social interaction. Recent developments in social neuroscience have revealed neural mechanisms by which such mutual adaptation can be realised. These mechanisms not only enable intentions to converge to an emerging common decision, but at the same time enable to achieve shared
\end{abstract}

\footnotetext{
Parts of the work described here were presented in a preliminary form $([3,30])$ as follows; this submission substantially extends this by giving more details of the approach and by providing additional steps of validation. Hoogendoorn, M., Treur, J., Wal, C.N. van der, and Wissen, A. van, Modelling the Interplay of Emotions, Beliefs and Intentions within Collective Decision Making Based on Insights from social neuroscience. In: Wong, K.K.W., Mendis, B.S.U., Bouzerdoum, A. (eds.), Proceedings of the 17th International Conference on Neural Information Processing, ICONIP'10. Lecture Notes in Artificial Intelligence, vol. 6443. Springer Verlag, 2010, pp. 196-206. Bosse, T., Hoogendoorn, M., Klein, M.C.A., Treur, J., and Wal, C.N. van der, Agent-Based Analysis of Patterns in Crowd Behaviour Involving Contagion of Mental States. Proc. of the 24th International Conference on Industrial, Engineering and Other Applications of Applied Intelligent Systems, IEA/AIE'11. Lecture Notes in Artificial Intelligence, Springer Verlag, 2011, in press. http://www.few.vu.nl/ wai/Papers/IEA11May4.pdf.
}

T. Bosse · M. Hoogendoorn · M. C. A. Klein · J. Treur $(\varangle) \cdot$ C. N. van der Wal · A. van Wissen Department of Artificial Intelligence, VU University, De Boelelaan, 1081 HV, Amsterdam,

The Netherlands

e-mail: treur@cs.vu.nl

URL: http://www.few.vu.nl/ treur

T. Bosse

e-mail: tbosse@few.vu.nl

URL: http://www.few.vu.nl/ tbosse

M. Hoogendoorn

e-mail: mhoogen@few.vu.nl

URL: http://www.few.vu.nl/ mhoogen

M. C. A. Klein

e-mail: mcaklein@few.vu.nl

URL: http://www.few.vu.nl/ mcaklein 
underlying individual beliefs and emotions. This paper presents a computational model for such processes. As an application of the model, an agent-based analysis was made of patterns in crowd behaviour, in particular to simulate a real-life incident that took place on May 4, 2010 in Amsterdam. From available video material and witness reports, useful empirical data were extracted. Similar patterns were achieved in simulations, whereby some of the parameters of the model were tuned to the case addressed, and most parameters were assigned default values. The results show the inclusion of contagion of belief, emotion, and intention states of agents results in better reproduction of the incident than non-inclusion.

Keywords Computational modelling - Collective decision making - Social neuroscience · Mirroring · Belief · Emotion · Intention · Crowd behaviour

\section{Introduction}

When it comes to group decision making versus individual decision making, it is often said that 'two heads are better than one', and 'the more the merrier'. Combining the individual capabilities in a group setting is often perceived as a benefit for all parties involved. However, deciding as a group comes with substantial challenges, as each group member has autonomous neurological processes, carrying, for example, private mental states such as emotions, beliefs, and intentions, which may seem hard to combine within a group. So, viewed from a distance, group decision making by reaching mutual agreement could be very hard. Yet, quite often coherent decisions are made by groups, and group members even seem to feel good with these decisions. In recent years, this seeming paradox has been resolved by developments in the new area called social neuroscience; e.g., [6,7,15,16,26].

The crux is that these private mental states are not so static and isolated as they may seem; they often show high extents of dynamics due to social interaction. In social neuroscience neural mechanisms have been discovered that indeed - often in unconscious manners - account for mutual mirroring effects between mental states of different persons; e.g., [33,39,45]. For example, an emotion expresses itself in a smile which, when observed by another person, automatically triggers certain preparation neurons (also called mirror neurons) for smiling within this other person, and consequently generates the same emotion. Similarly, mirroring of intentions and beliefs can be considered.

In this paper group decision making in stressful circumstances (with emergency evacuations as an example) is addressed. In these circumstances, emotions have an important interaction with the beliefs and intentions involved in a decision making process. The aim was to design a human-like computational model which is biological plausible by exploiting knowledge from social neuroscience about the relevant underlying mechanisms. Such a model may be useful not only for purposes of prediction, but also to obtain more insight in the dynamics of the social mechanisms and their emergent properties as described in a noncomputational manner in social neuroscience.

Based on findings from neuroscience (Sect. 2), the computational model ASCRIBE (for agent-based social contagion regarding intentions, beliefs and emotions) is introduced that

C. N. van der Wal

e-mail: cn.van.der.wal@few.vu.nl; c.n.vander.wal@vu.nl

URL: http://www.few.vu.nl/ cn.van.der.wal

A. van Wissen

e-mail: wissen@few.vu.nl; a.van.wissen@vu.nl

URL: http://www.few.vu.nl/ wissen 
not only incorporates mechanisms for mirroring emotions, intentions and beliefs between different persons (Sect. 3), but also addresses how within a person beliefs and emotions affect each other, and how they both affect the person's intentions (Sect. 4). A number of example simulations have been performed (Sect. 5).

As a case study the model was evaluated based on empirical data for crowd behaviour. Behavioural patterns emerging in large crowds are often difficult to regulate. Various examples have shown how things can easily get out of control when many people come together during big events. Especially within crowds, the consequences can be devastating when emotion spirals (e.g., for aggression or fear) develop to high levels. In Sects. 6-9, a computational analysis is presented of the incident that happened on Dam square in Amsterdam at the fourth of May in 2010, when large numbers gathered for the national remembrance of the dead ('dodenherdenking'). In the middle of a two minutes period of silence, one person started to shout, causing panic to occur among the people present. What happened there, as a result of a panic spiral, was a relatively mild incident in which 'only' a number of persons ended up in hospitals with fractures and bruises. The model ASCRIBE was extended to incorporate this crowd movement context (Sect. 7). To tune the latter model to specific characteristics, a specific automated parameter tuning method was used (Sect. 8). It is shown how the model is able to simulate this outburst of panic and its consequences (Sect. 9). Finally, the model is compared to an epidemiological model in Sect. 10, and Sect. 11 concludes the paper with a discussion.

\section{Background from social neuroscience}

As the aim was to obtain a biologically plausible human-like model, first some of the key concepts from social neuroscience are briefly reviewed. Within neuroscience it has been discovered that certain neurons have a mirroring function (e.g., [14,32,33,39,42-45]). In the context of the neural circuits in which they are embedded, these neurons show both a function in preparation for certain actions or bodily changes and a function to mirror similar states of other persons: they are active also when the person observes somebody else intending or performing the action or body change. This includes expressing emotions in body states, such as facial expressions. These neurons and the neural circuits in which they are embedded play an important role in social functioning (e.g., $[14,33,39,45])$. When mental states of other persons are mirrored by some of the person's own states, which at the same time play a role in generating their own behaviour, then this provides an effective basic mechanism for persons to fundamentally affect each other's mental states and behaviour. These discoveries are the basis for an exciting new research area, called social neuroscience.

A person's cognitive states usually induce emotions, as described by neurologist Damasio, $[12,13]$; for example:

'Even when we somewhat misuse the notion of feeling — as in "I feel I am right about this" or "I feel I cannot agree with you"-we are referring, at least vaguely, to the feeling that accompanies the idea of believing a certain fact or endorsing a certain view. This is because believing and endorsing cause a certain emotion to happen.' ([13], p. 93).

Damasio's Somatic Marker Hypothesis; cf. [1,10,11,13], is a theory on decision making which provides a central role to emotions felt. Within a given context, each represented decision option induces (via an emotional response) a feeling which is used to mark the option. For example, a strongly negative somatic marker linked to a particular option occurs as a strongly negative feeling for that option. Similarly, a positive somatic marker occurs as a positive feeling for that option ([10], pp. 173-174). 


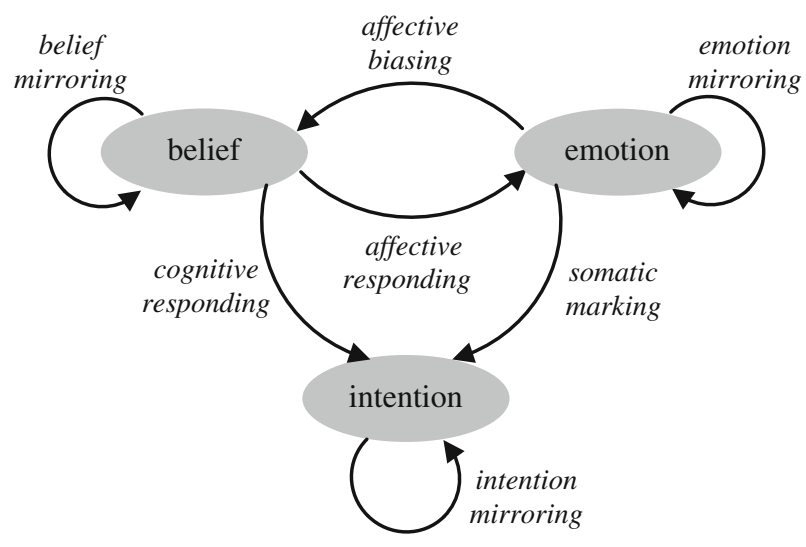

Fig. 1 The interplay of beliefs, emotions and intentions in social context

Table 1 Intra-agent and inter-agent interactions

\begin{tabular}{|c|c|c|c|}
\hline From & To & Agent & Description \\
\hline Belief & Emotion & Intra-agent & $\begin{array}{l}\text { Affective response on information; for } \\
\text { example, on threats and possibilities to } \\
\text { escape }\end{array}$ \\
\hline Emotion & Emotion & Inter-agent & $\begin{array}{l}\text { Emotion mirroring by nonverbal and verbal } \\
\text { interaction; for example, fear contagion }\end{array}$ \\
\hline Emotion & Belief & Intra-agent & $\begin{array}{l}\text { Affective biasing; for example, adapting } \\
\text { openness, amplification extent and } \\
\text { orientation }\end{array}$ \\
\hline Belief & Belief & Inter-agent & $\begin{array}{l}\text { Belief mirroring by nonverbal and verbal } \\
\text { interaction; for example, diffusion of } \\
\text { information on threats and possibilities to } \\
\text { escape }\end{array}$ \\
\hline Belief & Intention & Intra-agent & $\begin{array}{l}\text { Cognitive response on information; for } \\
\text { example, aiming for an exit that is believed } \\
\text { to be reachable }\end{array}$ \\
\hline Emotion & Intention & Intra-agent & $\begin{array}{l}\text { Somatic marking of intention options; for } \\
\text { example, giving options that feel bad a low } \\
\text { valuation }\end{array}$ \\
\hline Intention & Intention & Inter-agent & $\begin{array}{l}\text { Intention mirroring by nonverbal and verbal } \\
\text { interaction; for example, contagion of } \\
\text { tendency to go in a certain direction }\end{array}$ \\
\hline
\end{tabular}

In Fig. 1 an overview of the interplay of the different states within the model for collective decision making is shown.

It is assumed that at the individual level the strength of an intention for a certain decision option depends on the person's beliefs (cognitive responding) and emotions (somatic marking) in relation to that option. Moreover, it is assumed that beliefs may generate certain emotions (affective responding), for example fear, that in turn may affect the strength of beliefs (affective biasing). Note that it is assumed that these latter emotions are independent of the different decision options. Given this, to obtain collectiveness of the decision making a mirroring mechanism as briefly described above is used in three different ways; see also Fig. 1 and Table 1: 
- Mirroring of emotions is a mechanism for how fear and emotions felt in different individuals about a certain considered decision option mutually affect each other,

- Mirroring of beliefs is a mechanism transferring information on the extent to which different individuals believe certain information

- Mirroring of intentions is a mechanism transferring information between individuals on the strength of action tendencies (e.g., [23], p. 70) for certain decision options

These mechanisms describe not only how over time the individual decision intentions of group members may converge to a common group intention, but also how this relates to a basis of shared beliefs and shared emotions developed within the group at the same time. These shared belief and emotion states provide a solid grounding and robustness for the decisions. Indeed, the computational model introduced in Sects. 3 and 4 shows these types of patterns, as illustrated in Sect. 5.

\section{A computational model for mirroring of mental states}

A main building block of the computational model is a general model describing at an abstract level the mirroring of a given mental state $S$ (for example, an emotion, belief or intention). This is based upon the model that was also used as a generic building block in [29,31]. An important element is the contagion strength $\gamma_{S B A}$ from person $B$ to person $A$ in a group. This denotes how much the state $S$ of $A$ is influenced by the state $S$ of $B$. It is defined by

$$
\gamma_{S B A}=\varepsilon_{S B} \alpha_{S B A} \delta_{S A}
$$

Here, $\varepsilon_{S B}$ is the personal characteristic expressiveness of the sender $B$ for $S, \delta_{S A}$ the personal characteristic openness of the receiver $A$ for $S$, and $\alpha_{S B A}$ the interaction characteristic channel strength for $S$ from sender $B$ to receiver $A$. In order to determine the level $q_{S A}$ of state $S$ in an agent $A$, the following calculations are performed. First, the overall contagion strength $\gamma_{S A}$ from the group towards agent $A$ is calculated:

$$
\gamma_{S A}=\Sigma_{B \neq A} \gamma_{S B A}
$$

This value is used to determine the weighed impact $q_{S A}{ }^{*}$ of all the other agents upon state $S$ of agent $A$ :

$$
q_{S A}{ }^{*}(t)=\Sigma_{B \neq A} \gamma_{S B A} q_{S B}(t) / \gamma_{S A}
$$

The dynamics of the different mechanisms involved are modelled by dynamical relationships using the following general pattern:

$$
Y_{A}(t+\Delta t)=Y_{A}(t)+\gamma<\text { change_expression }>\Delta t
$$

Here the change of $Y$ is specified for a time interval between $t$ and $t+\Delta t$; the $\gamma$ represents the speed of the adjustment processes. Applied to the variable $q_{S A}(t)$ for $Y_{A}(t)$ the following is taken:

$$
<\text { change_expression }>=f\left(q_{S A}{ }^{*}(t), q_{S A}(t)\right)-q_{S A}(t)
$$

where $f\left(q_{S A}{ }^{*}(t), q_{S A}(t)\right)$ is a combination function. Therefore for the case considered:

$$
q_{S A}(t+\Delta t)=q_{S A}(t)+\gamma_{S A}\left[f\left(q_{S A}{ }^{*}(t), q_{S A}(t)\right)-q_{S A}(t)\right] \Delta t
$$

Two additional personal characteristics determine how much this external influence actually changes state $S$ of agent $A$, namely the tendency $\eta_{S A}$ to absorb or to amplify the level of 
Table 2 Parameters and states

\begin{tabular}{ll}
\hline$q_{S A}$ & Level for state $S$ for person $A$ \\
$\varepsilon_{S A}$ & Extent to which person $A$ expresses state $S$ \\
$\delta_{S A}$ & Extent to which person $A$ is open to state $S$ \\
$\eta_{S A}$ & Tendency of person $A$ to absorb or amplify state $S$ \\
$\beta_{S A}$ & Positive or negative bias of person $A$ on state $S$ \\
$\alpha_{S B A}$ & Channel strength for state $S$ from sender $B$ to receiver $A$ \\
$\gamma_{S B A}$ & Contagion strength for $S$ from sender $B$ to receiver $A$ \\
\hline
\end{tabular}

a state and the bias $\beta_{S A}$ towards increasing (upward) or reducing (downward) impact for the value of the state. Based on this the combination function $f\left(q_{S A}{ }^{*}(t), q_{S A}(t)\right)$ used was taken as:

$$
\begin{aligned}
f\left(q_{S A}{ }^{*}(t), q_{S A}(t)\right)= & \eta_{S A}\left[\beta_{S A}\left(1-\left(1-q_{S A}{ }^{*}(t)\right)\left(1-q_{S A}(t)\right)\right)\right. \\
& \left.+\left(1-\beta_{S A}\right) q_{S A}{ }^{*}(t) q_{S A}(t)\right]+\left(1-\eta_{S A}\right) q_{S A}{ }^{*}(t)
\end{aligned}
$$

By (4) the new value for the state $S$ at time $t+\Delta t$ is calculated from the old value at $t$, plus the change of the value based upon the transfer by mirroring. This change is defined as the multiplication of the overall contagion strength $\gamma_{S A}$ times the difference of a combination function of $q_{S A}{ }^{*}$ and $q_{S A}$ with $q_{S A}$. The combination function used has a component for amplification (after $\eta_{S A}(t)$ ) and one for absorption. The amplification component depends on the tendency of the person towards more positive (part multiplied by $\beta_{S A}(t)$ or negative (part of equation multiplied by $1-\beta_{S A}(t)$ side). Table 2 summarizes the most important parameters and states within this general model.

\section{Modelling the interplay of beliefs, emotions and intentions}

This section describes a computational model for the interplay of emotions, beliefs and intentions in a group of persons in the context of collective decision making. In this model the general model described in Sect. 3 is specialized for three different types of mental states $S$, namely beliefs, emotions, and intentions. In principle this is a large number of variants of Eq. (4) above for all persons $A$ in a group and all states $S$, indicated by belief $(X)$, fear, emotion $(O)$, intention $(O)$ for information $X$ and options $O$. However, in addition, at the individual level interactions between these different states are modelled, as depicted in Fig. 1; see also Table 1 for a brief explanation of all interactions in the model. This means that the model obtained by forming specializations of the generic model from Sect. 3 is modified in order to incorporate the internal interactions between the different types of states. For example, as can be seen in Table 3, the effect of beliefs on fear of a person has to be combined with the effect of fear of other group members on the own fear. This will be explained in more detail in the remainder of this section.

\subsection{The effect of emotions on beliefs}

To model the effect of emotions on information diffusion, below the personal characteristics $\delta_{S A}, \eta_{S A}$ and $\beta_{S A}$ for a belief state $S=\operatorname{belief}(X)$ are not assumed constant, but are instead modelled in a dynamic manner, depending on emotions. Personal characteristics $\varepsilon_{\text {belief }(X) A}, \delta_{\text {belief }(X) A}, \eta_{\text {belief }(X) A}, \beta_{\text {belief }(X) A}$ and interaction characteristic $\alpha_{\text {belief }(X) B A}$ are parameters in the model as described in Sect. 3. One additional category is introduced here, namely informational state characteristics $r_{X A}$ denoting how relevant, and $p_{X A}$ denot- 
Table 3 The different types of processes in the model

\begin{tabular}{|c|c|c|c|}
\hline From $S$ & To $S^{\prime}$ & Type & Description \\
\hline Belief $(X)$ & Fear & Internal & $\begin{array}{l}\text { Affective response on information; for } \\
\text { example, on threats and possibilities to } \\
\text { escape }\end{array}$ \\
\hline Emotion $(O)$ fear & Emotion $(O)$ fear & Interaction & $\begin{array}{l}\text { Emotion mirroring by nonverbal and verbal } \\
\text { interaction; for example, fear contagion }\end{array}$ \\
\hline Fear & Belief $(X)$ & Internal & $\begin{array}{l}\text { Affective biasing; for example, adapting } \\
\text { openness, amplification extent and } \\
\text { orientation }\end{array}$ \\
\hline Belief $(X)$ & Belief $(X)$ & Interaction & $\begin{array}{l}\text { Belief mirroring by nonverbal and verbal } \\
\text { interaction; for example, of information on } \\
\text { threats and options to escape }\end{array}$ \\
\hline Belief $(X)$ & Intention $(O)$ & Internal & $\begin{array}{l}\text { Cognitive response on information; for } \\
\text { example, aiming for an exit that is believed } \\
\text { to be reachable }\end{array}$ \\
\hline $\operatorname{Emotion}(O)$ & Intention $(O)$ & Internal & $\begin{array}{l}\text { Somatic marking of intention options; for } \\
\text { example, giving options that feel bad a low } \\
\text { valuation }\end{array}$ \\
\hline Intention $(O)$ & Intention $(O)$ & Interaction & $\begin{array}{l}\text { Intention mirroring by nonverbal and verbal } \\
\text { interaction; for example, of tendency to go } \\
\text { in a certain direction }\end{array}$ \\
\hline
\end{tabular}

ing how positive information $X$ is for person A. An assumption made for the model is that the intensity of the fear state of a person will affect his ability to receive information, by affecting the value of the individual person characteristics; in particular, a high level of fear affects $\beta_{\text {belief }(X) A}, \eta_{\text {belief }(X) A}$ and $\delta_{\text {belief }(X) A}$. First the effect of fear upon the openness for a belief belief $(X)$ (characterized by a relevance $r_{X A}$ of information $X$ for $A$ ) is expressed:

$$
\begin{aligned}
\delta_{\text {belief }(X) A}(t+\Delta t)= & \delta_{\text {belief }(X) A}(t)+\mu \cdot\left(1 / 1+e^{-\sigma\left(q_{\text {fear }, A}(t)-\tau\right)}\right) \\
& \cdot\left[\left(1-\left(1-r_{X A}\right) q_{\text {fear }, A}(t)\right) \delta_{\text {belief }(X) A}(t)\right] \cdot \Delta t
\end{aligned}
$$

If $q_{\text {fear }, A}$ is lower than threshold $\tau$ (on the interval [0,1]), it will not contribute to the value of $\delta_{\text {belief }(X) A}$. If $q_{\text {fear, } A}$ has a value above $\tau$, the openness will depend on the relevance of the information: when the relevance is high, openness will increase, while if the relevance is low, openness will decrease. In all formulae, $\mu$ is an adaptation parameter. This proposed model corresponds to theories of emotions as frames for selective processing, as described in [22,37]. A distinction between amplification values for different types of information is also made, depending on the emotional state fear. The dynamics for the characteristic $\eta_{\text {belief }(X) A}(t)$ modelling the amplification or absorption of $\operatorname{belief}(X)$ are described as follows:

$$
\begin{aligned}
\eta_{\text {belief }(X) A}(t+\Delta t)= & \eta_{\text {belief }(X) A}(t)+\mu \cdot\left(1 / 1+e^{-\sigma\left(q_{\text {fear }, A}(t)-\tau\right)}\right) \\
& \cdot\left[r_{X A} \cdot\left(1-p_{X A}\right) \cdot\left(q_{\text {fear }, A}(t) \eta_{\text {belief }(X) A}(t)\right)\right] \cdot \Delta t
\end{aligned}
$$

The emotion of fear only has an influence when it is above the threshold. In that case the parameter only changes for relevant, non-positive information for which the parameter starts to move towards the value for the emotion of fear (meaning this type of information will be amplified). This property represents an interpretation of [9] on how emotion can result in selective processing of emotion-relevant information. 
The bias of a person is also influenced by its emotion, but in addition depends on the content of the information, which can be either positive or negative:

$$
\begin{aligned}
\beta_{\text {belief }(X) A}(t+\Delta t)= & \beta_{\text {belief }(X) A}(t)+\mu \cdot\left(1 / 1+e^{\sigma\left(q_{\text {fear }, A}(t)-\tau\right)}\right) \cdot\left(1-q_{\text {belief }(X) A}(t)\right) \\
& \cdot\left[\left(\zeta_{A} \cdot p_{X A}+\left(1-\zeta_{A}\right) \cdot\left(1-p_{X A}\right)\right)-\beta_{\text {belief }(X) A}(t)\right] \cdot \Delta t
\end{aligned}
$$

Parameter $\tau$ is a number between 0 and 1 and represents a threshold for $q_{\text {fear }}$ : when $q_{\text {fear }}>$ $\tau$, then $q_{\text {fear, } A}$ has an influence on the bias $\beta_{\text {belief }(X) A}(t)$. Parameter $\zeta_{A}$ is a personality characteristic; if $\zeta_{A}=1$, represents a person who is optimistic when he/she has a lot of fear: positive information will be strengthened more and negative information will be weakened more. The reverse happens when $\zeta_{A}=0$, this represents a person who is more 'pessimistic' when experiencing fear: negative information will be strengthened and positive information will be weakened. Both personality characteristics seem to exist in people: a bias towards the negative side of information in case of experiencing a high level of fear, corresponds with the narrowing hypothesis from Frederickson's broaden-and-build theory in [21]. Others have a bias towards more positive information and emotions. Leaders could use this ability motivate their followers in times of crisis, as positive information and emotions broaden people's mindset [22], and focusing on positive information and emotions can contribute positively to individual's mental states (including attention and cognitive capacity) and resources [21]. The dynamically changing 'parameters' $\delta_{\text {belief }(X) A}(t), \eta_{\text {belief }(X) A}(t), \beta_{\text {belief }(X) A}(t)$ are used in the equation describing the dynamics of the belief state belief $(X)$ :

$$
\begin{aligned}
q_{\text {belief }(X) A}(t+\Delta t)= & q_{\text {belief }(X) A}(t)+\gamma_{\text {belief }(X) A}(t) \\
& \cdot\left[f\left(q_{\text {belief }}(X) A *(t), q_{\text {belief }}(X) A(t)\right)-q_{\text {belief }(X) A}(t)\right] \Delta t
\end{aligned}
$$

where the combination function $f\left(q_{S A}{ }^{*}(t), q_{S A}(t)\right)$ used is taken in a dynamic manner as:

$$
\begin{aligned}
& f\left(q_{\text {belief }(X) A^{*}(t), q_{\text {belief }}(X) A}(t)\right)=\eta_{\text {belief }}(X) A(t)\left[\beta_{\text {belief }}(X) A(t)\right. \\
& \cdot\left(1-\left(1-q_{\text {belief }(X) A}{ }^{*}(t)\right)\left(1-q_{\text {belief }(X) A}(t)\right)\right) \\
& \left.+\left(1-\beta_{\text {belief }(X) A}(t)\right) q_{\text {belief }(X) A}{ }^{*}(t) q_{\text {belief }(X) A}(t)\right] \\
& +\left(1-\eta_{\text {belief }(X) A}(t)\right) q_{\text {belief }(X) A}{ }^{*}(t)
\end{aligned}
$$

Note that since it depends on $\delta_{\text {belief }(X) A}(t)$, also $\gamma_{\text {belief }(X) A}(t)$ becomes dynamic.

\subsection{The effect of beliefs on emotions in the dynamics of fear}

Besides modelling the influence of emotion upon the information contagion in the previous Section, the opposite direction is investigated in this Section: emotions being influenced by information. This influence is modelled by altering the overall weighed impact of the contagion of the emotional state for fear. This is expressed as follows:

$$
\begin{aligned}
q_{\text {fear }, A}{ }^{*}(t)= & v_{A} \cdot\left(\sum_{B \neq A} \gamma_{\text {fear } B A} \cdot q_{\text {fear } B} / \gamma_{\text {fear } A}\right) \\
& +\left(1-v_{A}\right) \cdot\left(\sum_{X} \omega_{X, \text { fear }, A} \cdot\left(1-p_{X A}\right) \cdot r_{X A} \cdot q_{\text {belief }(X) A}\right)
\end{aligned}
$$

Here the influence depends on the impact from the emotion fear by others (the first factor, with weight $v_{A}$ ) in combination with the influence of the belief present within the person. In this case, information has an increasing effect on fear if it is relevant and non positive. This $q_{f e a r, A^{*}}(t)$ is used in the equation describing the dynamics of fear:

$$
q_{\text {fear } A}(t+\Delta t)=q_{\text {fear } A}(t)+\gamma_{f e a r A}\left[f\left(q_{\text {fear } A} *(t), q_{\text {fear } A}(t)\right)-q_{\text {fear } A}(t)\right] \Delta t
$$


with

$$
\begin{aligned}
f\left(q_{\text {fear } A}{ }^{*}(t), q_{\text {fear } A}(t)\right)= & \eta_{\text {fear } A}\left[\beta_{\text {fear } A}\left(1-\left(1-q_{\text {fear }}{ }^{*}(t)\right)\left(1-q_{\text {fear } A}(t)\right)\right)\right. \\
& \left.+\left(1-\beta_{\text {fear } A}\right) q_{S A}{ }^{*}(t) q_{S A}(t)\right] \\
& +\left(1-\eta_{\text {fear } A}\right) q_{\text {fear } A}{ }^{*}(t)
\end{aligned}
$$

\subsection{The effects of beliefs and emotions on intentions}

The abstract model for mirroring described above applies to emotion, belief and intention states $S$ for an option $O$ or the situation in general, but does not describe any interplay for intentions yet. Taking the Somatic Marker Hypothesis on decision making as a point of departure, not only intentions of others, but also own emotions affect the own intentions. To incorporate such an interaction, the basic model is extended as follows: to update $q_{\text {intention }(O) A}$ for an intention state $S$ relating to an option $O$, both the intention states of others for $O$ and the $q_{\text {emotion }}(O) A(t)$ values for the emotion state $S^{\prime}$ for $O$ are taken into account. These intention and emotion states $S$ and $S^{\prime}$ for option $O$ are denoted by $O I$ and $O E$, respectively:

Level of fear of person $A$ :

Level of emotion for option $O$ of person $A$ :

$q_{\text {fearA }}(t)$

$q_{\text {emotion }(O) A}(t)$

qintention $(O) A(t)$

qbeliefsfor $(O) A(t)$

Here $q_{\text {beliefsfor }(O) A}(t)$ denotes to aggregated support for option $O$ by beliefs of $A$; it is defined as

$$
q_{\text {beliefsfor }(O) A}(t)=\sum_{X} \omega_{X O A} q_{\text {belief }(X) A} / \sum_{X} \omega_{X O A}
$$

where $\omega_{X O A}$ indicates how supportive information $X$ is for option $O$. The combination of the own (positive) emotion level and the rest of the group's aggregated intention is made by a weighted average of the two:

$$
\begin{aligned}
q_{\text {intention }(O) A^{* *}(t)=} & \left(\omega_{O I A 1} / \omega_{O I E B A}\right) q_{\text {intention }(O) A}{ }^{*}(t) \\
& +\left(\omega_{O E A 2} / \omega_{O I E B A}\right) q_{\text {emotion }(O) A}(t) \\
& +\left(\omega_{O B A 2} / \omega_{O I E B A}\right) q_{\text {beliefsfor }}(O) A \\
& (t) \\
\gamma_{\text {intention }(O) A^{*}=} & \omega_{O I E B A} \gamma_{\text {intention }(O) A}
\end{aligned}
$$

where $\omega_{O I A 1}, \omega_{O B A 2}$ and $\omega_{O E A 2}$ are the weights for the contributions of the group intention impact (by mirroring), the own emotion impact (by somatic marking), and the own belief impact on the intention of $A$ for $O$, respectively, and

$$
\omega_{O I E B A}=\omega_{O I A 1}+\omega_{O E A 2}+\omega_{O B A 2}
$$

The combination of the own belief level and the rest of the group's aggregated emotion for a certain option $\mathrm{O}$ is made by a weighted average of the two

$$
\begin{aligned}
& q_{\text {emotion }(O) A^{* *}}(t)=\left(\omega_{O E A 1} / \omega_{O E B A}\right) q_{\text {emotion }}(O) A^{*}(t) \\
& +\left(\omega_{O B A 1} / \omega_{O E B A}\right) q_{\text {beliefsfor }(O) A}(t) \\
& \gamma_{\text {emotion }(O) A}{ }^{*}=\omega_{O E B A} \gamma_{\text {emotion }}(O) A
\end{aligned}
$$

where $\omega_{O E A 1}$ and $\omega_{O B A 1}$ are the weights for the contributions of the group emotion impact (by mirroring), the own belief impact on the emotion of $A$ for $O$, respectively, and $\omega_{O E B A}=$ 
$\omega_{O E A 1}+\omega_{O B A 1}$. Then the overall model for the dynamics of emotions and intentions for options becomes:

$$
\begin{aligned}
& q_{\text {emotion }(O) A}(t+\Delta t)=q_{\text {emotion }}(O) A(t)+\gamma_{\text {intention }}(O) A^{*} \\
& \cdot\left[\eta _ { \text { emotion } ( O ) A } \left(\beta _ { \text { emotion } ( O ) A } \left(1-\left(1-q_{\text {emotion }(O) A^{* *}}(t)\right)\right.\right.\right. \\
& \left.\left(1-q_{\text {emotion }(O) A}(t)\right)\right) \\
& \left.+\left(1-\beta_{\text {emotion }(O) A}\right) q_{\text {emotion }(O) A}{ }^{* *}(t) q_{\text {emotion }(O) A}(t)\right) \\
& \left.+\left(1-\eta_{\text {emotion }(O) A}\right) q_{\text {emotion }(O) A}{ }^{* *}(t)-q_{\text {emotion }(O) A}(t)\right] \cdot \Delta t \\
& \left(1-\left(1-q_{\text {intention }}(O) A^{* *}(t)\right)\left(1-q_{\text {intention }}(O) A(t)\right)\right) \\
& \left.+\left(1-\beta_{\text {intention }(O) A}\right) q_{\text {intention }(O) A}{ }^{* *}(t) q_{\text {intention }(O) A}(t)\right) \\
& \left.+\left(1-\eta_{\text {intention }(O) A}\right) q_{\text {intention }(O) A}{ }^{* *}(t)-q_{\text {intention }(O) A}(t)\right] \cdot \Delta t
\end{aligned}
$$

\section{Some example simulation results for a fictional case study}

In this section, some example results of a small fictional case study will be presented. The goal of the case study was to investigate if the computational model can simulate the interplay of emotions, intentions and beliefs, as described in neuroscientific, social and psychological literature. The computational model was implemented in Matlab in the context of an evacuation scenario (see Appendix $\mathrm{A}^{1}$ for the complete Matlab specification).

The example scenario is expressed as follows: at the end of a working day in an office, the fire alarm goes off and all the persons that are in the building need to evacuate immediately. At the time of the alarm, 3 teams of each 3 people are present on different floors, as can be seen in Fig. 2. Persons can communicate with each other when they are on the same floor, or they can communicate to each other through their personal device, which is equipped with a tool for sharing emergency information over a short distance. Communication through such personal devices can only occur in case the distance is three floors or less. The building has four emergency exits, three on the ground floor and one on the fifth floor via a skyway to another building. If an exit is accessible, the information is rated as 'positive' information in the model, if not accessible then the information is rated 'not positive'. In the formalization, this leads to the following information state characteristics: $p_{E x i t}=1$ for accessible exits and $p_{E x i t X}=0$ for blocked exists. The relevance of this information for survival is always one, i.e. $r_{E x i t X}=1$.

\subsection{An example scenario}

In the example scenario, the three persons located on the top floor know that exit four is available (i.e. they have a belief of one in information $p_{\text {Exit } 4}=1$ ), whereas the three persons on the middle floor do not have any strong beliefs about any of the emergency exits. The three on the first floor know the situation of the exits one and two at the first floor, thus they have beliefs of strength one concerning those exists. In this case, the first exit is blocked and

\footnotetext{
${ }_{1}^{1}$ http://www.cs.vu.nl/ mhoogen/social-diffusion/AppendixA-ICONIP10.pdf.
} 


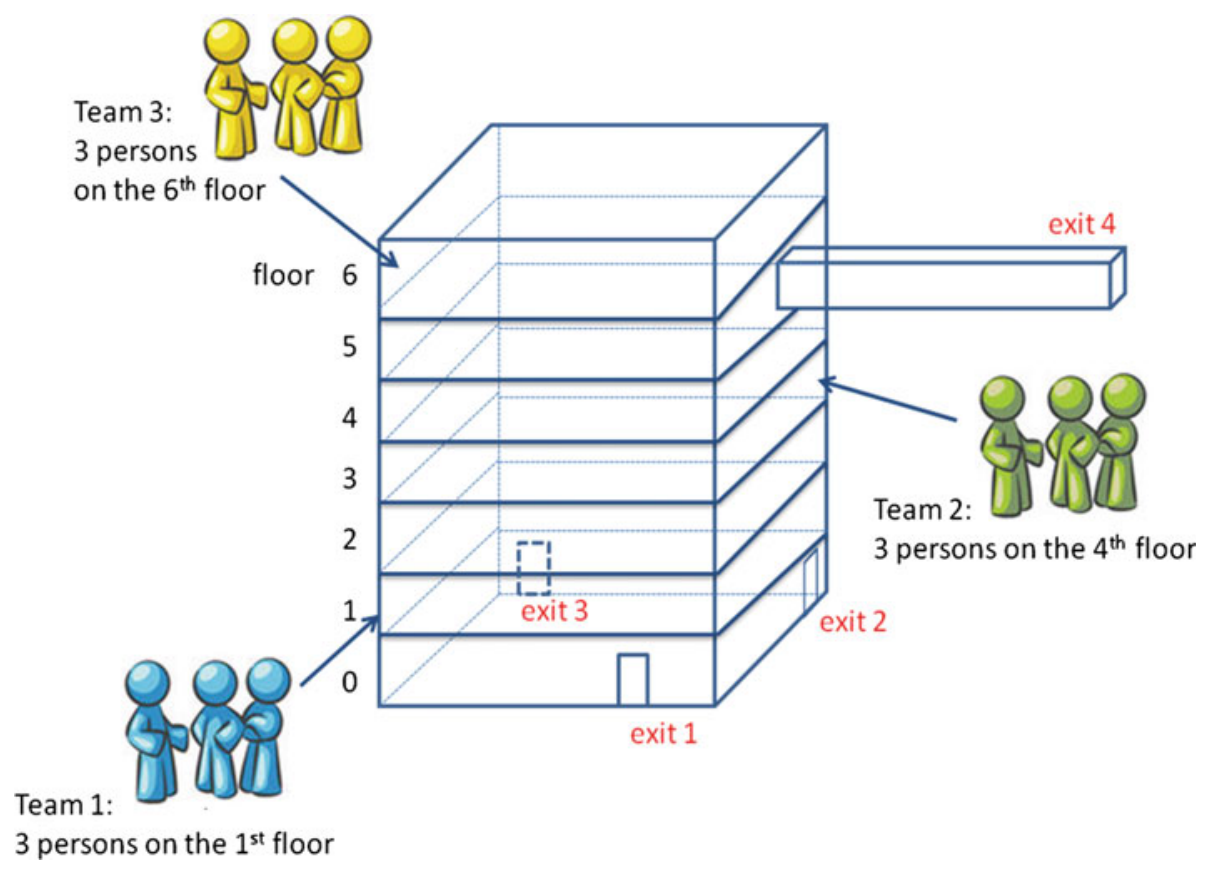

Fig. 2 The location of three teams in a building of six floors with four exits

the second is accessible, therefore $p_{\text {Exit } 1}=0$ and $p_{\text {Exit } 2}=1$. They do not know anything about exit three, therefore a belief of strength 0 is present concerning exit three. Besides these values, all other values are set to 0.5 with respect to the beliefs to indicate that they know the exits are there but do not know specifically whether the exit is accessible or not. Moreover, the intentions of all agents are initially set to zero (i.e. they start with no specific intention to leave the building via any of the exits) and the emotions to $0,1,0$, and 1 for exit 1, 2, 3, and 4 respectively (since exit one and exit three represent negative information, the emotion for that option is not positive). Finally, for the emotion of fear the agents on the first floor have no fear, on the middle floor they have maximum fear, and on the top floor medium fear is present. Furthermore, the initial belief about the situation itself is 0.5. Regarding all the parameter settings as described before: each agent has the same initial set of parameters, and these can be found in the Matlab specification as shown in Appendix A.

Figure 3 shows the change of the values of the beliefs, intentions, and emotions. The top four rows represent the values related to the four exits. Here, the values for all agents during the simulation runs are shown. The $y$-axis of the graphs represents all nine persons, who have values for certain variables, stated on the $z$-axis. The values develop over time, which is represented by the $x$-axis. At the bottom row of the figure, diagrams with the amount of fear and the judgment of the entire situation are shown. It can be seen that fear spreads quickly, resulting in a very negative judgment of the situation by all agents. For exit one the belief about the exit being an option for evacuation eventually stabilizes at a relatively low value due to the fact that no human has a good feeling for that option (although in the beginning the emotions are slightly pulled upwards as well as the intention, due to the very strong belief of the three agents at the first floor). For exits two and four a very strong belief occurs rapidly for all agents as well as a very strong intention and the positive emotions also remain high. 

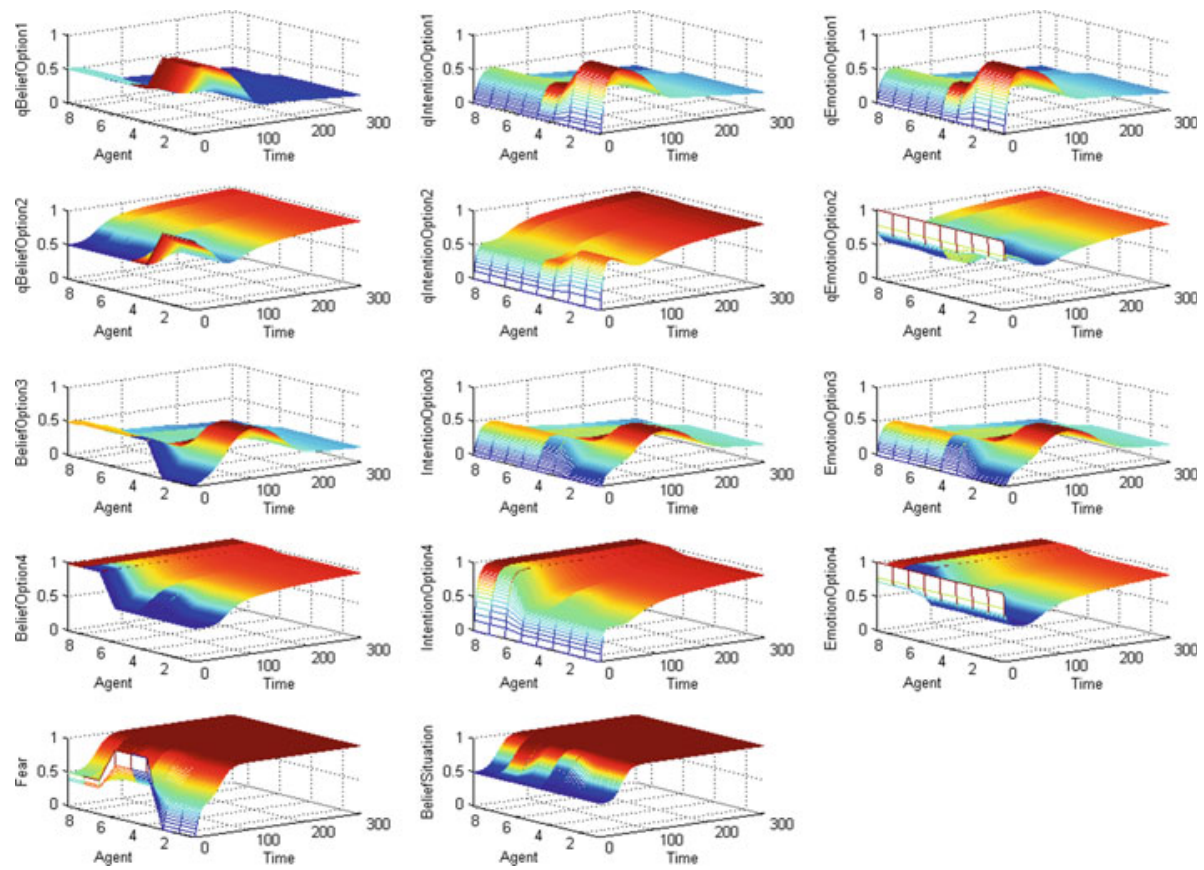

Fig. 3 Simulation results for an example scenario

Finally, for exit three the agents on the first floor get a slightly stronger belief, intention, and emotion due to the fact that the other agents have a belief with value 0.5 about the exit. Eventually however, the values return to a rather low value again due to the fact that the others have lowered their value again. Without the ability to communicate with each other using personal devices, the beliefs, intentions, and emotions would not have been influenced by those on the other floors.

\subsection{More systematic variations}

The context of this case study was used to explore whether under a variety of parameter settings patterns emerge as expected.

\subsubsection{The effect of information on fear}

A first prediction about the interplay of emotions, intentions and beliefs, according to the computational model is derived from formula (11): it is expected that if a person experiences a situation as dangerous, then this persons's fear level should increase. Simulations where the persons believed that the situation is dangerous were compared with simulations where they believed that that situation was not dangerous. The result of these simulations were that if persons believe that the situation is not dangerous $\left(p_{\text {belief }}(s) A=1\right)$, then $q_{\text {fear } A}(t)$ goes to 0 , meaning that the persons will experience no fear. If the persons believe that the situation is dangerous $\left(p_{\text {belief }(s) A}=0\right)$, then $q_{\text {fear } A}(t)$ increases to 1 , meaning that the persons will increase their experience of fear, when they consider the situation as dangerous. This result corresponds with our expectation. 


\subsubsection{The effect of emotion on beliefs}

According to formulas (6), (7), and (8) the level of fear that a person is experiencing, can have an effect on the way a person processes information. More precisely: it is expected that when $q_{\text {fear } A}(t)$ is above threshold $\tau$, then the emotion fear should have an effect on the way persons process information. Multiple simulations were run to test this. In the simulations, the threshold $\tau$ was set to 0.5 and the initial value of $q_{\text {fear } A}(t)$ is below or above threshold $\tau$, for example, 0.1 or 0.7 . Whenever $q_{\text {fear } A}(t)$ is above the threshold $\tau$ (either from the start, or at a later time point), $\delta_{\text {belief }(X) A}(t), \eta_{\text {belief }(X) A}(t)$ and $\beta_{\text {belief }(X) A}(t)$ start to change indeed. Here results will be briefly presented where $\zeta$ was one.

- The openness $\delta_{\text {belief }(X) A}(t)$ becomes one or stays one, this is according to the model, because when $\zeta=1$ and $r_{\text {belief }(s) A}=1$ (the information is relevant for survival), $\delta_{\text {belief }(X) A}(t)$ should increase.

- The bias factor $\beta_{\text {belief }(X) A}(t)$ increases for the situation, exit one and three (which are not accessible), but decreases for exit two and four (which are accessible). This is what was expected, because the higher $p_{\text {belief }(s) A}$ is (meaning the more 'positive' information is), the lower $\beta_{\text {belief }(X) A}(t)$ should become (meaning information will be spread weaker by this person), the lower $p_{\text {belief }}(s) A$ is, the higher $\beta_{\text {belief }(X) A}(t)$ should become (meaning strengthening the spread of negative information).

- The amplification extent $\eta_{\text {belief }(X) A}(t)$ increases differently for the situation, where exit 1 and 3 are not accessible. For this situation it goes towards one and it increases more, the further the agents are away from the exit. This is according to expectation, because $\eta_{\text {belief }(X) A}(t)$ should only increase if $p_{\text {belief }(s) A}=$ low and $r_{\text {belief }}(s) A=$ high, in these instances, $p_{\text {belief }(s) A}=0$ and $r_{\text {belief }(s) A}=1$. For exits two and four, $p_{\text {belief }(s) A}=1$ and $r_{\text {belief }(s) A}=1$. In that case $\eta_{\text {belief }}(X) A(t)$ should not increase, and that is what is happening correctly in this evacuation scenario.

\subsubsection{The effects of a combination of beliefs and emotions}

In the simulations it was found that the combination of emotions and beliefs decreases the level of $q_{\text {emotion }(X) A}(t)$ more than they do separately. This effect was expected from formula (17) for $q_{\text {emotion }(X) A}(t)^{* *}$. For example, here one can see that in this situation the combination of emotions and beliefs makes $q_{\text {emotion }(X) A}(t)$ increase more, than when beliefs are not combined with emotions.

\section{A real world case study: the May fourth incident}

The computational model mentioned above was applied to the May fourth incident in Amsterdam (The Netherlands). The incident took place in the evening of May fourth 2010, when approximately 20,000 people gathered on Dam Square in Amsterdam for the National Remembrance of the dead. What follows is a short description of the events.

At 20:00 h everyone in the Netherlands, including the crowd on Dam Square, was silent for two minutes to remember the dead. Fences and officials compartmented the 20.000 people on Dam Square. At 20:01 a man in the crowd on Dam Square disturbed the silence by screaming loudly. People standing directly near him could see that this man looked a bit 'crazy' or 'lost', and they did not move. Those not within a few meters of the screaming source, started to panic and ran away from the man that screamed. The panic spread through the people that 
were running away who infected each other with their emotions and intentions to flee. This panic was fuelled by a loud 'BANG' that was heard about three seconds after the man started screaming. Queen Beatrix and other royal members present were escorted to a safe location nearby. In total, 64 persons got injured: they got broken bones and scrapes by being pushed, or got run over by the crowd. The police exported the screaming man and got control over the situation within two minutes. After $2 \frac{1}{2} 2$ minutes, the master of ceremony announced to the crowd that a person had become ill and had received care. He asked everybody to take his or her initial place again, and to continue the ceremony. After this, the ceremony continued.

Eyewitness reports were collected on site. Below, parts of their transcripts are freely translated in English:

\section{Question: describe what happened in your own words.}

Eyewitness 1: "[...] The moment people in front of me started to run, I panicked. I could not see why these people ran away. It seemed they were running away from something. I immediately thought back to what happened the year before in Apeldoorn [the witness refers to the failed attack on the Royal Family the year before, when someone drove his car into the crowd towards the Royal Family and killed eight people]. After the yelling, I heard a lot of noise, which later turned out to be fences that fell down... [...] The few seconds when people started to run away after the scream, I found the most scary."

Eyewitness 2: "[...] The moment the man started screaming, I 'choked' of fear: I thought that someone wanted to disrupt the ceremony with an attack and I was afraid that we, including our little daughter, would be run over."

Eyewitness 3: [...] "My panic was growing when people behind us ran into the fences in 'blind panic'. The moment I saw fences falling down en people tripping over them was the peak of my panic. After that, I felt shocked and surprised, because people shouted 'Not again?!'. We helped people get back up on their feet and after that I fell into the arms of my friends and tried to relax. Because of the adrenaline, I could not manage easily. I had a bad feeling about the applause after the message of the master of ceremony: the message that somebody became ill and was taken care off, was clearly a lie and the applause was out of place."

\section{Question: how did you interpret the scream?}

Eyewitness 1: "Like an emergency call or yell of somebody with the wrong intentions." Eyewitness 2: "As a suicide terrorist who braces himself before he will act."

Eyewitness 3: "As a tortured soul that lost somebody and could only express this by screaming."

\section{Question: did you get information about the situation from people around you?}

Eyewitness 1: "No."

Eyewitness 2: "No, only that everybody was scared."

Eyewitness 3: "People, police and veterans were running everywhere. All attention was focused on the wounded and on recovering the peace. No information came to us. “

A short movie with images from the live broadcast on Dutch National Television, can be found at: http://www. youtube.com/watch?v=0cEQp8OQj2Y. This shows how, within two minutes, the crowd starts to panic and move. 


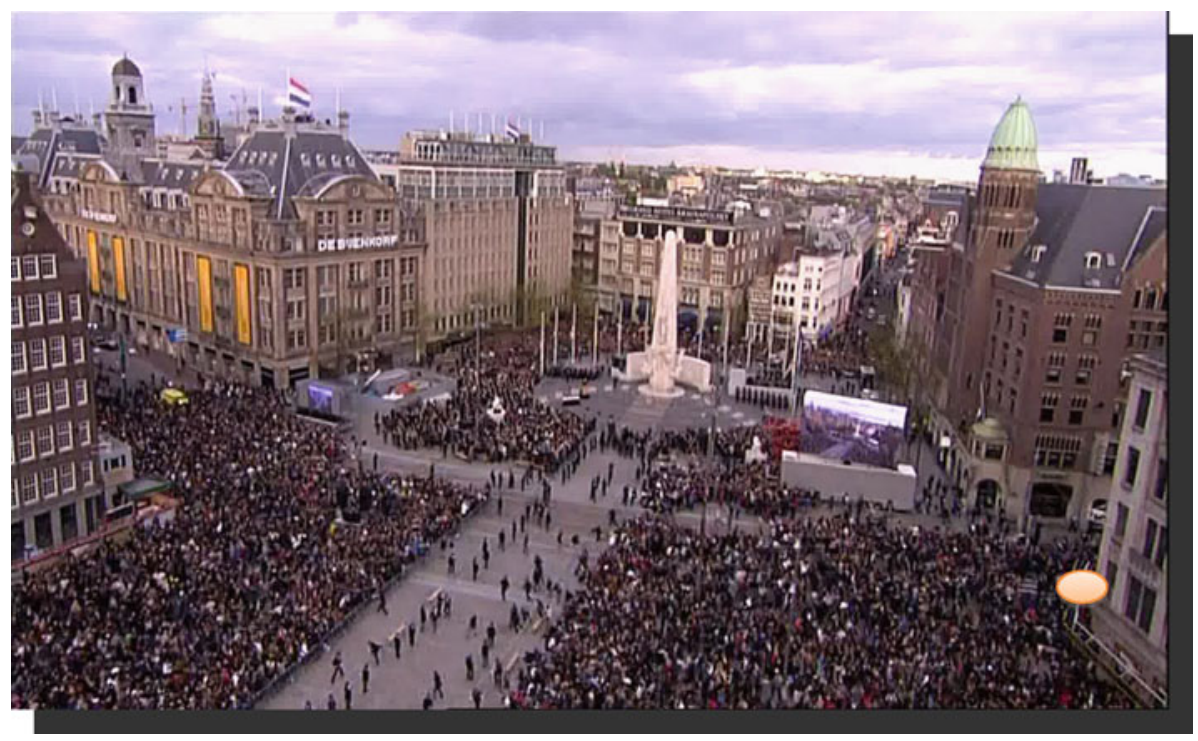

Fig. 4 Still image of the people on Dam Square starting to flee. The circle on the right bottom indicates the location of the yelling person

The live broadcast of the National Remembrance on Dutch National Television has been acquired in HD-quality.In this video, one can see the crowd on Dam Square flee from the perspective shown in Fig. 4. The video includes the cuts and editing that were done during the live broadcast, because the un-edited video material of all cameras that were filming that day was not saved.

From the total broadcast, a shorter three minutes movie was made, starting the moment when the crowd was silent and the person started to scream loudly. In this three minutes movie there are two time slots that were further processed (11-17 and 20-27 s), because (i) they showed the clear camera angle like the one that can be seen in Fig. 4, and (ii) the direction and speed of the movements of people could be clearly analysed. They were analysed as follows. The movie was cut into still images, to detect the location of people by hand. Ten still images per second were chosen in order to be able to detect the movements of running people frame by frame. By keeping track of the coordinates of mouse-clicks on the locations of people in the crowd while they were moving, their trace of movement could be detected.

A total of 130 frames were analysed by hand. Not all people could be analysed, both because of the quantity, and the impossibility to trace every 'dot' (person) over multiple still images. Persons in different positions of the crowd with simultaneous movements to the people around them were chosen, such that these target subjects were able to represent multiple people around them. In total 35 persons were traced. See Fig. 5, for an example of five of the persons that were traced. The red dot represents the screaming man. The five blue lines represent the five persons that were traced. The arrows indicate in which direction the persons ran away. The $x$ - and $y$-axis contain the coordinates.

The density of the crowd around a target subject was also acquired, which could be used to build a representative large-scale simulation consisting of ten thousands of agents. Since the exact number of persons surrounding a target could not be distinguished in the video,

Permission granted for educational and research purposes by The Netherlands Institute for Sound and Vision. 


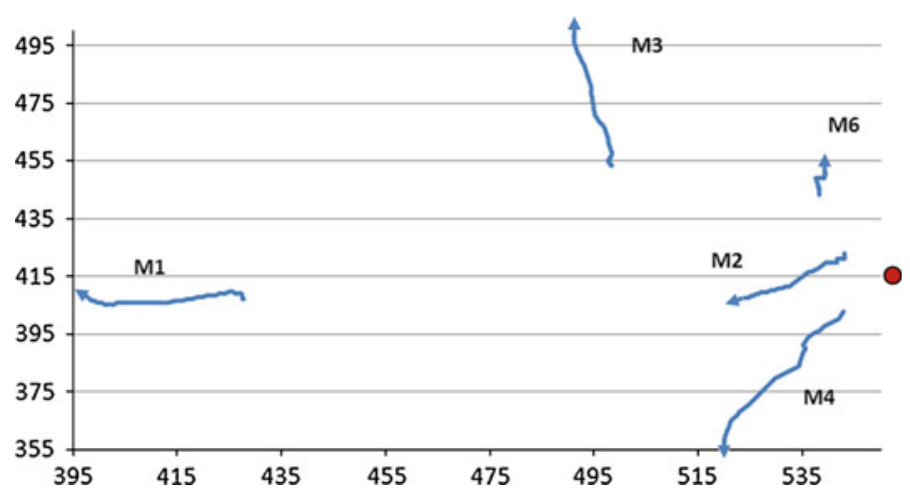

Fig. 5 Escape directions of five persons that were traced by hand

three distinctions in density were made: high, medium and low. The size of the circle around the target subject, in which density was measured, is shown on the right in Fig. 4.

The next step was to correct for the angle the camera makes with the floor by recalculating the coordinates into coordinates that would fit into a bird's-eye view on the Dam Square, perpendicular to the floor. People's distances in meters from corners of the buildings were translated to the position in pixels on a $600 \times 800$ map of the area, using offsets and scaling. Specifically, the following formulae are used to translate movements in pixels to movements in meters:

$$
\begin{aligned}
& x_{\text {meter }}=x_{\text {pixel }} / 22 \\
& y_{\text {meter }}=y_{\text {pixel }} / 8
\end{aligned}
$$

This was then transformed to the map using the following formulae:

$$
\begin{aligned}
& x_{\text {map }}=\left(x_{\text {meter }} * 5.15\right)+136 \\
& y_{\text {map }}=\left(y_{\text {meter }} * 5.15\right)-167
\end{aligned}
$$

The bird's eye view perspective used in the computational model can be seen in Fig. 6. The resulting figure was represented in the simulation in Matlab. Locations of certain obstacles, like buildings and fences, were also transformed into the bird's-eye view.

\section{Extending and specialising the model ASCRIBE for the May fourth case}

To tailor the model ASCRIBE towards the domain introduced in Sect. 6, a number of steps were taken.

\subsection{Case specific states}

First of all, the relevant states for the agents have been distinguished. In this case, the emotion, belief and intention states relate to the options for each agent. A total of nine options are available including 'remain standing', and moving in any wind direction (N, NE, E, SE, $\mathrm{S}, \mathrm{SW}, \mathrm{W}, \mathrm{NW}$ ). Besides these, there is an additional belief about the current situation. This expresses how positive a person judges the current situation (zero a negative judgment, and 


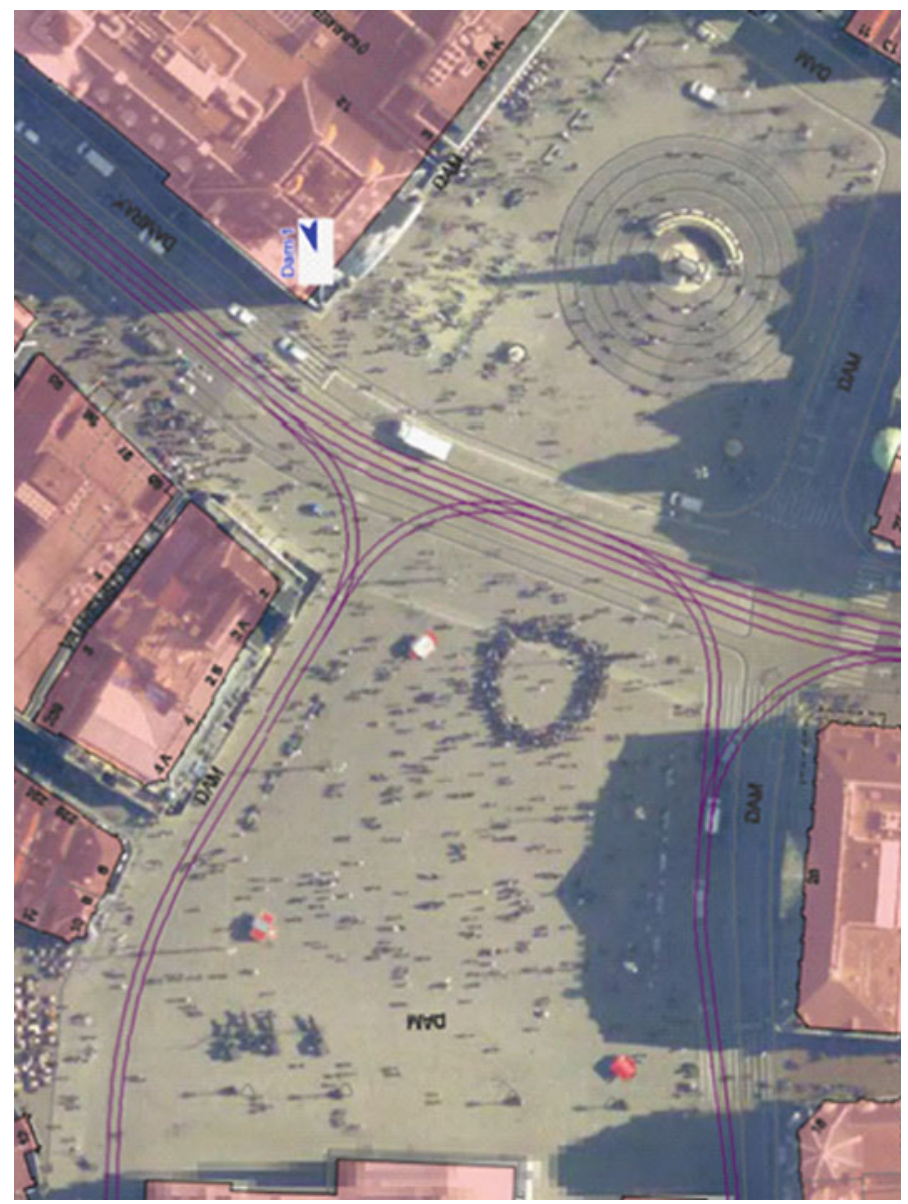

Fig. $6600 \times 800$ pixel image of the Dam Square

one a positive judgment). Finally, the emotions for each option and the emotion fear are represented.

\subsection{Channel strength}

In the scenario described above, the channel strengths between the various agents are dependent on the physical location of the agents. If other agents are close, the channel strength is high, whereas it is low or zero in case agents are far apart. Therefore, a threshold function was used expressing within which reach agents still influence each other in a significant manner:

$$
\alpha_{S B A}(t)=1-\left(1 / 1+e^{-\sigma(\text { distance }} B A^{\left.(t)-\tau_{\text {distance }}\right)}\right)
$$

Here $\sigma$ and $\tau_{\text {distance }}$ are global parameters and distance $_{B A}$ is the Euclidean distance between the positions $\left(x_{A}(t), y_{A}(t)\right)$ and $\left(x_{B}(t), y_{B}(t)\right)$ of $A$ and $B$ at $t$. 


\subsection{Movement}

The movement of the agents directly depends upon their intentions. Recall that the strength of the intention is determined by the intentions of others (see Sect. 3), and the agent's own personality characteristics and mental states, such as beliefs and emotions (see Sect. 4). The highest feasible intention is selected (in cases where certain movements are obstructed, the next highest intention is selected). For each of the selected options $O$, the movement $x_{\text {movement }(O)}$ on the $x$-axis and $y_{\text {movement }}(O)$ on the $y$-axis is specified; e.g., the option for going south means -1 step on the $y$-axis and none on the $x$-axis: $x_{\text {movement }}(O)=0$ and $y_{\text {movement }(O)}=-1$. The actual point to which the agent will move is then calculated by taking the previous point and adding the movement of the agent during a certain period to that. The movement of the agent depends upon the strength of the intention for the selected option and the maximum speed with which the agent can move. If the intention is maximal (i.e., 1) the agent will move with the maximum speed. In case the intention is minimal (i.e., 0 ) the agent will not move. The dependency between mental states and speed of movement has been described in several works. It has long been acknowledged, starting with the intuitive 'fight or flight' concept, that emotions prime the human body for action ([19,23, 24,34]). Emotions are considered to fall into two categories; those that elicit approach responses and those that elicit withdrawal responses. It has been noted that both fear and disgust often include behavioural components of withdrawal (e.g., [20,40]). Unpleasant cues activate the defensive system (i.e., danger, fear), which facilitates movements away from the cue (e.g., $[8,47])$. Ekman claims that in case of fear withdrawal entails escaping from the threatening stimulus. Quantarelli states more specifically that "panic is marked by loss of self-control, that is, by unchecked fear, being expressed in flight" and that "panic most frequently takes the form of actual physical running" ([41], p.272, 269). In particular, a positive correlation between the emotion of fear and intensity of escape attempts was found (except in extremely stressful situations, in which impairment seemed to occur) (cf. [25]). This acknowledges a theory laid down in [35] that "it is generally assumed that level of fear is related positively to response in a potential panic situation". Given these underlying theories, the model that establishes this relationship is expressed as follows:

$$
\begin{aligned}
& x_{A}(t+\Delta t)=x_{A}(t)+\text { max_speed }_{A} \cdot q_{\text {intention }(O) A}(t) \cdot x_{\text {movement }}(O) \cdot \Delta t \\
& y_{A}(t+\Delta t)=y_{A}(t)+\text { max_speed }_{A} \cdot q_{\text {intention }}(O) A(t) \cdot y_{\text {movement }}(O) \cdot \Delta t
\end{aligned}
$$

Here the maximum speeds max_speed A $_{A}$ are agent-specific parameters.

\section{The parameter tuning method used}

As explained above, the computational model contains a large number of parameters; these parameters address various aspects of the agents involved, including their personality characteristics (e.g., expressiveness, openness, and tendency to absorb or amplify mental states), physical properties (e.g., minimum and maximum speed, and limit of their sight), and characteristics of their mutual interactions (e.g., channel strength between sender and receiver). The accuracy of the model (i.e., its ability to reproduce the real world data as closely as possible) heavily depends on the settings of these parameters. Therefore, parameter estimation techniques [50] have been applied to learn the optimal values for the parameters involved.

In order to determine what is 'optimal', first an error measure needs to be defined. The main goal is to reproduce the movements of the people involved in the scenario; thus it was 
decided to take the average (Euclidean) distance (over all agents and time points) between the actual and simulated location:

$\varepsilon=\sum_{\text {agents }} \sum_{\text {a timepoints } t} \frac{\sqrt{(x(a, t, \operatorname{sim})-x(a, t, \text { data }))^{2}+(y(a, t, \operatorname{sim})-y(a, t, \text { data }))^{2}}}{\text { \#agents } \cdot \# \text { timepoints }}$

Here, $x(a, t, \operatorname{sim})$ is the $x$-coordinate of agent $a$ at time point $t$ in the simulation, and $x(a, t$, data) the same in the real data (similarly the $y$-coordinates). Both are in meters.

Next, the relevant parameters were tuned to reduce this error. To this end, the approach described in detail in Sects. 3 and 4 of [4] was used. This approach makes use of the notion of sensitivity of variables for certain parameter changes. Roughly spoken, for a given set of parameter settings, the idea is to make small changes in one of the parameters involved, and to observe how such a change influences the change of the variable of interest (in this case the error). Here, 'observing' means running the simulation twice, i.e., once with the original parameter settings, and once with the same settings were one parameter has slightly changed. Formally, the sensitivity $S_{X, P}$ of changes $\Delta X$ in a variable $X$ to changes $\Delta P$ in a parameter $P$ is defined as follows (note that this sensitivity is in fact the partial derivative $\partial X / \partial P): S_{X, P}=\Delta X / \Delta P$. Based on this notion of sensitivity, the adaptation process as a whole, is an iterative process, which roughly consists of: (1) calculating sensitivities for all parameters under consideration, and (2) using these sensitivities to calculate new values for all parameters. This second step is done by changing each parameter with a certain amount $\Delta P$, which is determined as follows: $\Delta P=-\lambda * \Delta X / S_{X, P}$. Here, $\Delta X$ is the deviation found between actual and simulated value of variable $X$, and $\lambda$ is a speed factor. Note that, since in the current case $X$ represents the error, the 'actual value' of $X$ is of course 0 , so $\Delta X$ simply equals $\varepsilon$ in the simulation.

\section{Results}

This section presents the results of specialising and tuning the agent-based model with 35 agents, to the real world data of the May fourth incident. The results are presented for the first part of the data (i.e., seconds 11-17 of the three minutes movie). To assess the performance of the model, it was compared with three other models, which are introduced in Sect. 9.1. Next, Sect. 9.2 explains which parameters of those models were tuned, and which parameter settings were found. Sect. 9.3 discusses the results of running the models for the optimal parameter settings found; in particular, for each model the increase of the error over time (of the simulation) is shown. Sect. 9.4 discusses the statistical significance of the results, and Sect. 9.5 illustrates the behaviour of the simulation based on the optimal models found.

\subsection{Models used for comparison}

To assess the performance of the ASCRIBE model, it was compared to three other models. First, one baseline model was developed in which the agents do not move at all. Second, the model was compared to an implementation of the model by Helbing et al. [27], which is currently one of the most influential models in the area of crowd simulation. This model has been specifically designed for simulating dynamical features of escape panic, and is essentially a specific variant of a social force model for pedestrian dynamics which Helbing and Molnar introduced in 1995 [28]. It has been modelled following the framework of self-driven many particle systems and is based on a general force model. The model assumes that each agent 
likes to move in a certain direction with a certain desired velocity. In addition however, the agent is influenced by certain interaction forces: it wants to keep a certain distance from other agents and walls. The model is expressed by means of a number of equations (cf. [27]), and a very brief overview of the main equations in the model is presented here. For a complete overview of the model, see [27]. In the first equation the change in the velocity of the agent is given as follows:

$$
m_{i} \frac{\mathrm{d} \mathbf{v}_{\mathrm{i}}}{\mathrm{d} t}=m_{i} \frac{v_{i}^{0}(t) \mathbf{e}_{i}^{0}(t)-\mathbf{v}_{i}(t)}{\tau_{i}}+\sum_{j(\neq i)} \mathbf{f}_{i j}+\sum_{W} \mathbf{f}_{i W}
$$

This expresses that the velocity of the agent changes based upon the desired velocity $\left(v_{i}^{0}(t)\right)$, the desired direction $\left(\mathbf{e}_{i}^{0}(t)\right)$, and the current velocity. Note that in the implementation of the model, a more sophisticated variant of the desired direction has been used (cf. [28]) in which a complete set of points to be visited can be expressed, and the desired direction depends on the closest of these points given the current position. The forces that occur due to other agents and walls are added to the equation as well. In the equation, the parameter $m_{i}$ represents the mass of the agent, whereas $\tau_{i}$ expresses the so-called characteristic time. In order to calculate the forces from other agents and walls, two equations are used. The first of these equations concerns the calculation of the force between an agent $\mathrm{i}$ and $\mathrm{j}$ :

$$
\mathbf{f}_{i j}=\left\{A_{i} \exp \left[\left(r_{i j}-d_{i j}\right) / B_{i}\right]+k g\left(r_{i j}-d_{i j}\right)\right\} \mathbf{n}_{i j}+\kappa g\left(r_{i j}-d_{i j}\right) \Delta v_{j i}^{t} t_{i j}
$$

The equation indicates that the force between two agents is dependent upon a number of factors. The first part of the equation $\left(\left\{A_{i} \exp \left[\left(r_{i j}-d_{i j}\right) / B_{i}\right]+k g\left(r_{i j}-d_{i j}\right)\right\} \mathbf{n}_{i j}\right)$ expresses the body force between the agents, where $A_{i}, B_{i}$, and $k$ are constants. Furthermore, $d_{i j}$ represents the distance between the two agents, $\mathbf{n}_{i j}$ is the normalized vector pointing from agent $\mathrm{j}$ to agent $\mathrm{i}$, and $r_{i j}$ is the sum of the change of positions of agent $\mathrm{i}$ and $\mathrm{j} . g\left(r_{i j}-d_{i j}\right)$ evaluates to zero in case the pedestrians do not touch each other (i.e. $d_{i j}>r_{i j}$ ) and otherwise to $r_{i j}-d_{i j}$. The second part of the equation $\left(\kappa g\left(r_{i j}-d_{i j}\right) \Delta v_{j i}^{t} t_{i j}\right)$ represents the so-called sliding friction in case the pedestrians come close to each other. Here, $\Delta v_{j i}^{t}$ is the tangential velocity difference, and $t_{i j}$ the tangential direction.

The second equation concerns the interaction with walls and is quite similar to the equation used for the interaction with other agents:

$$
\mathbf{f}_{i W}=\left\{A_{i} \exp \left[\left(r_{i}-d_{i W}\right) / B_{i}\right]+k g\left(r_{i}-d_{i W}\right)\right\} \mathbf{n}_{i W}-\kappa g\left(r_{i}-d_{i W}\right)\left(\mathbf{v}_{i} \mathbf{t}_{i W}\right) \mathbf{t}_{i W}
$$

In this case, $d_{i W}$ is the distance between the agent and the wall, and $\mathbf{n}_{i W}$ is the direction perpendicular to the wall. Furthermore, $\mathbf{t}_{i W}$ is the direction tangential to the wall.

Finally, next to the no motion model and the Helbing et al. model, a variant of ASCRIBE was developed in which all agents also make individual decisions, but do not influence each other (i.e., no contagion takes place). This was done to assess whether the idea and implementation of contagion of mental states is useful at all.

This resulted in three different models (in addition to our own ASCRIBE model with contagion of mental states), to which we refer below as baseline, Helbing, and without contagion, respectively. To enable a fair comparison, parameter tuning was applied for all models (except for the baseline model, since it did not contain any parameters to tune) in order to find optimal settings, as explained in the next section. 
Table 4 Optimal parameter settings found

\begin{tabular}{|c|c|c|c|c|c|c|c|}
\hline \multicolumn{2}{|c|}{$\begin{array}{l}\text { Global parameters } \\
\text { (not tuned) }\end{array}$} & \multicolumn{2}{|c|}{$\begin{array}{l}\text { Initial variable } \\
\text { settings (not tuned) }\end{array}$} & \multicolumn{2}{|c|}{$\begin{array}{l}\text { Global parameters } \\
\text { (tuned) }\end{array}$} & \multicolumn{2}{|l|}{$\begin{array}{l}\text { Initial variables } \\
\text { (tuned) }\end{array}$} \\
\hline \#Agents & 35 & $\varepsilon_{\text {Intention }}$ & 0.5 & $\tau$ Distance & 190 & qBelief (nomove) & 0.005 \\
\hline Max_x & 600 & $\delta_{\text {Intention }}$ & 0.5 & Sight_reach & 200 & & \\
\hline Max_y & 800 & $\eta_{\text {Intention }}$ & 0.5 & Max_speed & Differs per agent & & \\
\hline$\Delta \mathrm{t}$ & 0.5 & $\beta$ Intention & 0.5 & & & & \\
\hline$\mu_{\delta}$ Belief & 0.5 & $\varepsilon_{\text {Belief }}$ & 0.5 & & & & \\
\hline$\mu_{\eta \text { Belief }}$ & 0.5 & $\delta$ Belief & 0.5 & & & & \\
\hline$\mu_{\beta \text { Belief }}$ & 0.5 & $\eta_{\text {Belief }}$ & 0.5 & & & & \\
\hline SBelief & 0.5 & $\beta$ Belief & 0.5 & & & & \\
\hline$\sigma$ & 100 & $\varepsilon_{\text {Emotion }}$ & 0.5 & & & & \\
\hline$\omega_{\text {OIA } 1}$ & 0.3 & $\delta$ Emotion & 0.5 & & & & \\
\hline$\omega_{\mathrm{OEA}} 2$ & 0.3 & $\eta_{\text {Emotion }}$ & 0.5 & & & & \\
\hline$\omega_{\mathrm{OBA} 2}$ & 0.3 & $\beta$ Emotion & 0.5 & & & & \\
\hline$\omega_{\text {OEA1 }}$ & 0.5 & & & & & & \\
\hline$\omega_{\mathrm{OBA} 1}$ & 0.5 & & & & & & \\
\hline All qbelief(X) & 0 & & & & & & \\
\hline $\begin{array}{l}\text { Impact of } \\
\text { event on }\end{array}$ & 1 & & & & & & \\
\hline $\begin{array}{r}\text { qbelief(X) } \\
\text { Min_speed }\end{array}$ & 0.01 & & & & & & \\
\hline
\end{tabular}

\subsection{Parameter settings}

The number of parameters to tune for the full ASCRIBE model is large; therefore, before starting the tuning process for this model, the settings for a large majority of the parameters were fixed at default values (see Table 4). For example, parameters with a relatively small sensitivity were left out of consideration for the tuning process (cf. [4]). For these parameters, reasonable default settings were chosen by hand (based on experimentation). The values of the remaining parameters (among others, the maximum speed for each individual agent, the minimum distance within which agents influence each other, and the initial values of one of the beliefs, see Table 4) were initialised by hand, but were then adapted using the parameter tuning approach described in the previous section.

The speed factor $\lambda$ of this tuning process was set to 0.1 . The initial locations of the agents involved were taken equal to the locations in the real world data. An overview of all optimal settings found for the global parameters and the initial variables involved in the model is shown in Table 4.

Here, the settings shown in the first two columns were set by hand, and the settings shown in the last two columns were found after tuning. Note that all settings (except those for maximum speed) were used globally for all agents.

For the model without contagion, the tuned parameters were the same as for the full model with contagion. For the Helbing model, the parameters that were tuned were also the desired speed for all individual agents $\left(v_{i}^{0}(t)\right)$, as well as the global parameters characteristic time $\left(\tau_{i}\right)$ and the difference between the points to be visited (representing the path the agent want to follow). Moreover, for the parameters $A, B, k$ and $\kappa$, the settings as prescribed in their article [27] were taken and as desired direction $\mathbf{e}_{i}^{0}(t)$ the direction precisely opposite to 


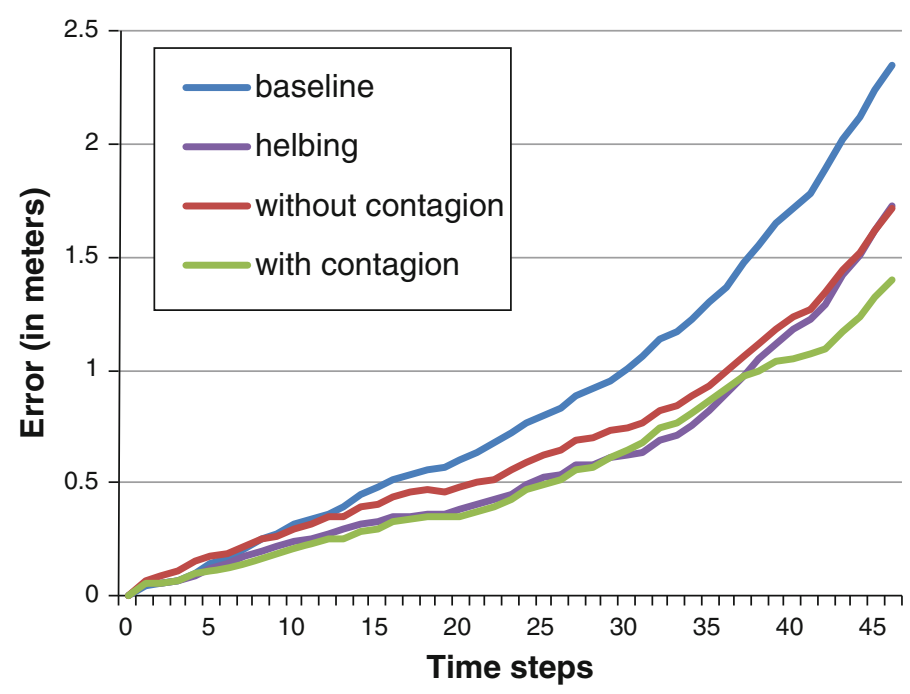

Fig. 7 Development of error over the simulation for four variants of the model. Each time step corresponds to a video frame, which were processed every $0.1 \mathrm{~s}$

the location of the shouting man was selected, and points were generated which form a path away from this location (thereby setting the desired direction to the closest point as explained before). The distance between these points depended on the setting of the parameter. For all models, the tuning was continued until the improvements made per iteration were smaller than $0.1 \%$.

\subsection{Increase of error over time}

Figure 7 shows for each of the four variants how the average error (over all agents) increases during the simulation.

Note that the error is expressed in meters. At the first time point, the error is zero (all agents start at their actual position), but over time the error increases very quickly in the baseline case, so that the error at the last time step of the simulation becomes quite large $(2.35 \mathrm{~m})$. For this model, the average error per time step is $0.87 \mathrm{~m}$. The average error found for the tuned model without contagion is much lower (0.66, i.e., an improvement of $24 \%$ ), and is even lower for the tuned model with contagion (0.54, i.e., an improvement of $38 \%$ ). This finding provides evidence for the conclusion that incorporating the contagion makes the model more accurate, even when it is based on default settings for the parameters. Note that in the current scenario, the agents' movements involve relatively small steps, compared to the size of the grid; the total distance that the agents travel during the 7 seconds of analysis is only $2.35 \mathrm{~m}$. Therefore, the relative errors found (i.e., the percentages of improvement mentioned above) are more insightful that the absolute errors. In case the total distance travelled would have been larger, the absolute difference in performance between the four models would be expected to have been bigger as well.

As for the Helbing model, the average error of this model per time step was found to be 0.59 (i.e., an improvement of $32 \%$ w.r.t. the baseline model). As can be observed from Fig. 7 , this model performs better than the model without contagion, but worse than the model with contagion (at least, in this particular scenario). One of the main reasons for this is that the 
model with contagion seems to be better able to deal with the fact that some agents only start moving half way the scenario. This phenomenon, which is also well visible in the video of the event, is caused by the fact that the crowd is separated by fences (see also Fig. 4), and especially the people that are located on the left hand side of the area wait a couple of seconds before they start moving, whereas other people start moving right after the scream. In the model with contagion, this phenomenon can be reproduced quite accurately by means of the contagion mechanism: the agents at the left hand side of the area initially have a low level of fear (since they are not directly affected by the screaming man), but only when they observe other agents panicking and trying to escape, they are influenced by them and attempt to get away as well. Since the Helbing model does not include an explicit mechanism for contagion of mental states, it has more difficulties in reproducing this particular effect (because in this model, the speed by which the agents move is more stable - although not completely constant-over time). Therefore, for the Helbing model, the parameter tuning resulted in an optimal situation where some agents on the left hand side hardly move at all. This is reflected by the fact that the error for this model (compared to the model with contagion) only increases in the last eight time steps.

When comparing the Helbing model with the model without emotion, one can observe that, although the errors of both models at time point 45 are comparable, the Helbing model performs slightly better when taking the overall average error over all time points. This can in part be explained by the fact that the Helbing model has more freedom when it comes to selecting the direction in which the agents move. In our model (both with and without contagion), selection of actions has been implemented in such a way that the agents can only pick one out of eight wind directions (see Sect. 7), whereas the Helbing model uses a continuous scale for this. We speculate that the performance of our model (both with and without contagion) may be further improved by changing this discrete mechanism for action selection into a continuous mechanism.

In order to provide some more insight in the variance of the error over the 35 agents, additional graphs have been generated which show the standard error of the mean $(\sigma / \sqrt{n}$, i.e., the standard deviation divided by the square root of the number of agents) at each time point for all models; see Fig. 8. These graphs show that the standard error is relatively small, which implies that errors are fairly distributed over the 35 agents, although there are some outliers (between 1 and 2.5 standard deviations), see next section. They also show that the standard error is largest for the baseline model, and smallest for the model with contagion.

\subsection{Statistical analysis}

A two-way within-subjects analysis of variance was conducted to evaluate the effect of the type of computational crowd behaviour model on deviation from the walking direction in the real world scenario. The analysis has been conducted for all time points of the simulation, for 35 agents. The dependent variable was the deviation from the walking direction in the real world scenario data named 'error', which was measured in meters. The within-subjects factors were type of crowd behaviour models, with four levels (Baseline, Helbing, ASCRIBE model without contagion, ASCRIBE model with contagion) and time with 47 levels (47 time steps of the simulations. Note that the models are deterministic, so that the source of variation comes from the agent population in each model and not from different runs of each model. The question that is investigated is whether the movement patterns of the agent population differs for each model, using the same initial values, but different contagion and influence mechanisms. The main effects and interactions were tested using the univariate 

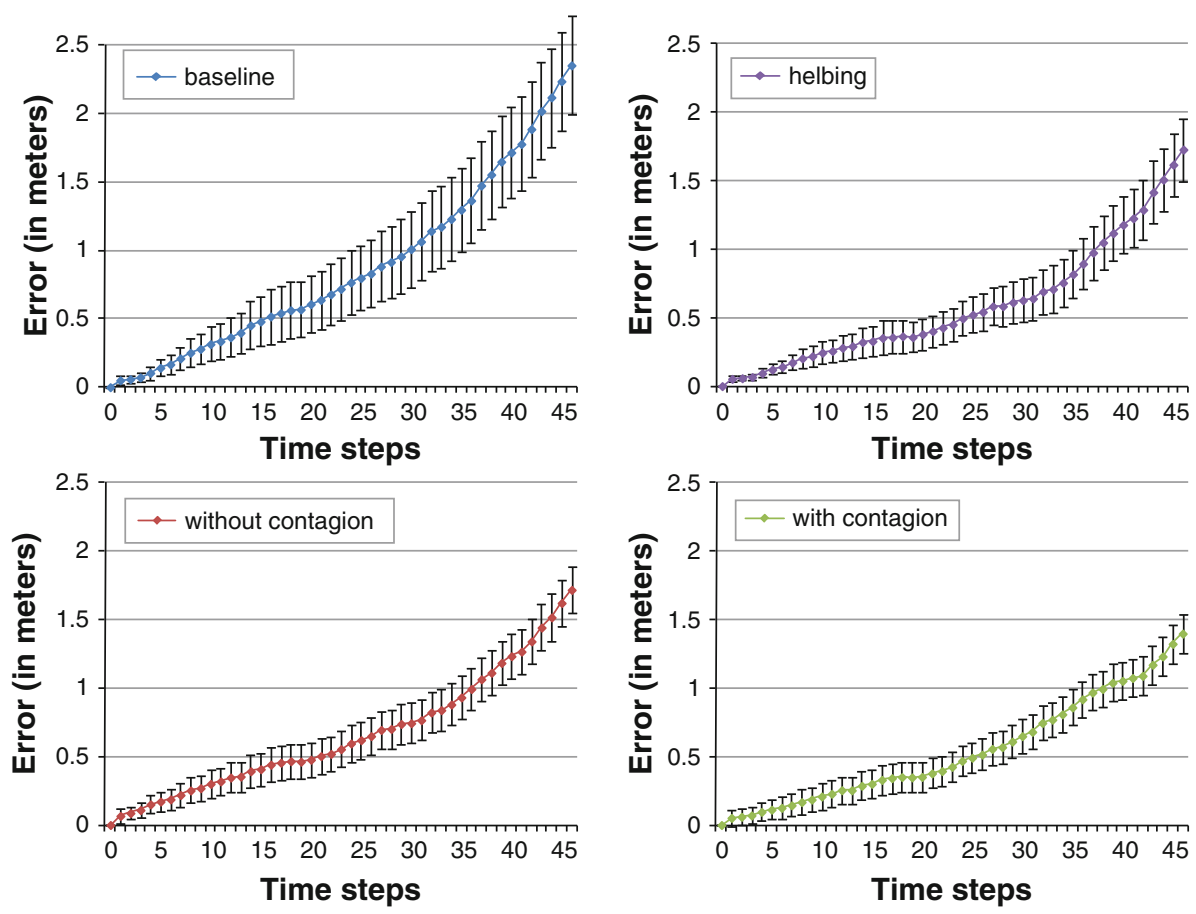

Fig. 8 Standard error at each time point for the four models

Huyhnh-Feldt analysis that corrects for non-sphericity. The Model main effect was significant, $F(1.4,48.2)=5.7, p<0.012$, the Time main effect was significant, $F(1.3,45.6)=26.1$, $p=0.012$, and the ModelxTime interaction effect was significant, $F(2.5,87.5)=6.7, p=0.001$. To further inspect which models differ significantly from each other, each combination of two models was tested with post hoc pairwise comparisons with LSD adjustment for significance on the $p<0.05$ level. Significant differences were found between Baseline and Helbing, $p<0.05$, Baseline and ASCRIBE with contagion, $p<0.05$, ASCRIBE without contagion and ASCRIBE with contagion, $p<0.001$, and a trend was found between Baseline and ASCRIBE without contagion, $p=0.074$. No significant difference was found between ASCRIBE with contagion and Helbing, $p=0.322$ and between Helbing and ASCRIBE without contagion, $p=0.171$.

These results seem to point in the direction that all models differ significantly, except Helbing from ASCRIBE with contagion and Helbing from ASCRIBE without contagion. Indeed, in Fig. 7, Helbing and ASCRIBE without contagion do seem to behave alike, but Helbing and ASCRIBE with contagion seem to differ substantially. We feel that when there would be more timesteps available in the real world data, the Helbing model would differ significantly from ASCRIBE with contagion, and perhaps even from ASCRIBE without contagion. Furthermore, when investigating the data further, two outliers can be found that differ between one and two standard deviations of the other 33 agents. (In Fig. 9 it can be seen that each model always has minimum one or two agents that behave very differently from the rest). When these two outliers are removed, all previously found significant differences stay significant (on the $p<0.01$ level), the difference between Helbing and ASCRIBE without contagion is still not significant, $p=0.38$, and a trend becomes visible between Helbing and 

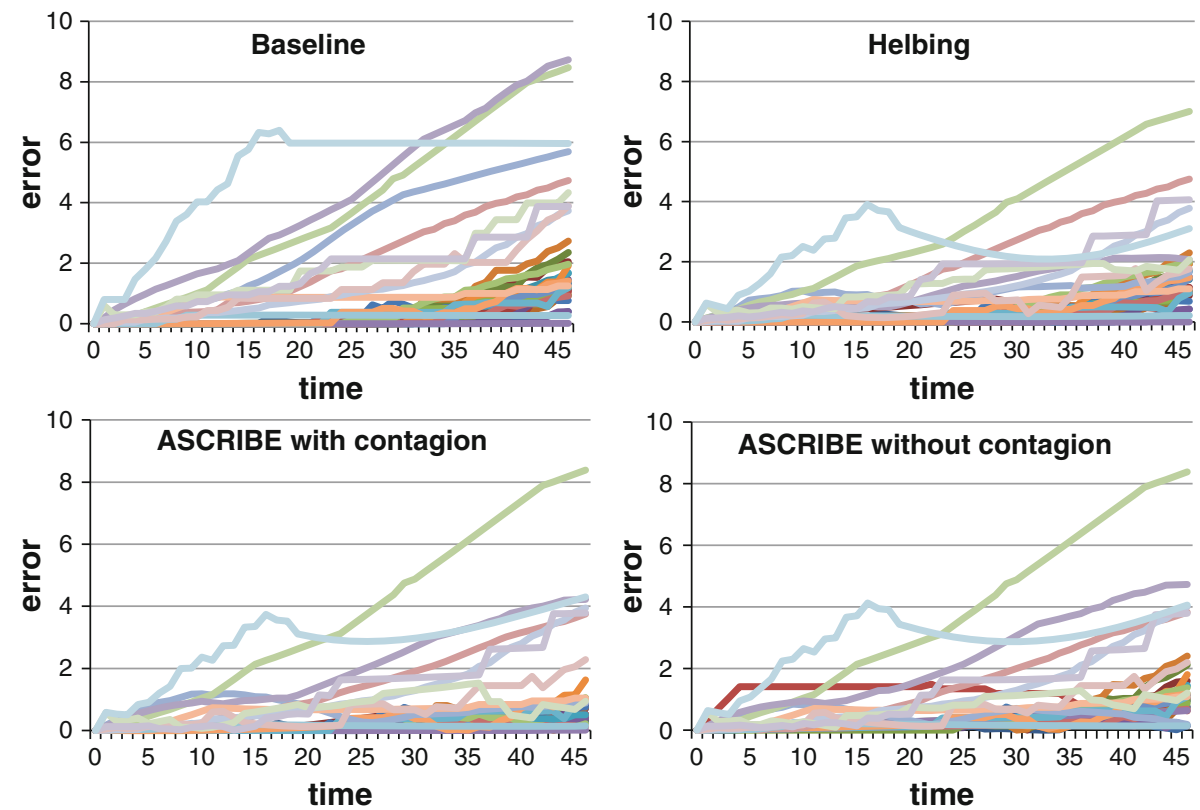

Fig. 9 Individual agent's error per model

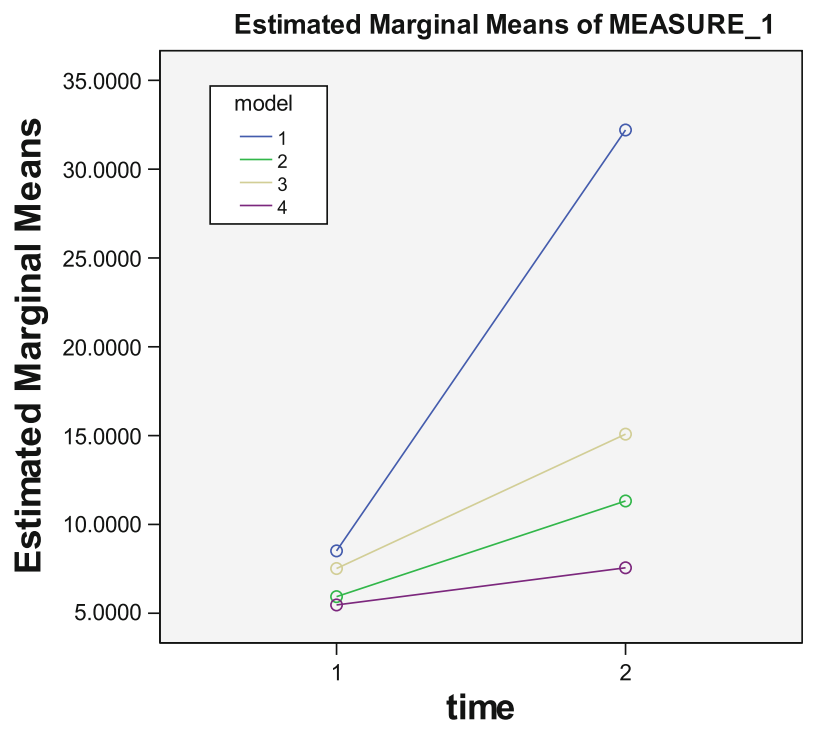

Fig. 10 Summed errors for all agents, per model, per half of simulation

ASCRIBE with contagion, $p=0.054$. These results point into the direction that all models differ significantly from each other in this scenario, except Helbing and ASCRIBE without contagion.

A second research question to be analysed statistically is: do the four computational models differ significantly on the second half of the simulation, compared to the first half 
of the simulation. This research question stems from the fact that in the second half of the simulation the whole mass of people is moving, compared to the first half of the simulation, where only the right half of the mass starts to move. In this way, the data has two distinct time points that can be compared, by summing up all data points per model, per half of the simulation. A two-way within-subjects analysis of variance was conducted to evaluate the effect of type of computational crowd behaviour model on deviation from the walking direction in the real world scenario on two time points: the first and second half of the simulation. See Fig. 10 for an overview of the summed errors per model, per time step. The within-subjects factors were type of crowd behaviour model with four levels (Baseline, Helbing, ASCRIBE model without contagion, ASCRIBE model with contagion) and time with two levels (the summed first half and second half of the simulation). The main effects and interactions were tested using the univariate Huyhnh-Feldt analysis that corrects for non-sphericity. The model main effect was significant, $F(1,35.3)=8.9$, $p=0.005$, the time main effect was significant, $F(1,34)=77.9, p<0.001$, and the model $\times$ time interaction effect was significant, $F(1.1,36.2)=10.5, p=0.002$. To further inspect which models differ significantly from each other, each combination of two models was tested with post hoc pairwise comparisons with LSD adjustment for significance on the $p<0.05$ level. Significant differences were found between all models: Baseline and Helbing, $p<0.01$, Baseline and ASCRIBE without contagion, $p<0.05$, Baseline and ASCRIBE with contagion, $p<0.001$, Helbing and ASCRIBE without contagion, $p<0.001$, Helbing and ASCRIBE with contagion, $p<0.001$, ASCRIBE without contagion and ASCRIBE with contagion, $p<0.001$.

\subsection{Resulting behaviour of the simulation}

After the tuning process was finished, the optimal settings found for all parameters were used as input for the four simulation models, to generate simulation traces which closely resemble the real world scenario. Using visualisation software (written in Matlab), these simulation traces have been visualised in the form of a 2D animation A screenshot of the animation of the model with contagion is shown in Fig. 11.

Here, the lines represent fences that were used to control the crowd, the large circle represents the monument on the square (see Fig. 4 for the actual situation), and the big dots represent corners of other buildings. The plus sign on the right indicates the location of the screaming man. The small dots represent the actual locations of the 35 people in the crowd that were tracked, and the stars represent the locations of the corresponding agents in the simulation. Even at the end of the simulation (see Fig. 11), the distances between the real and simulated positions are fairly small for this model.

\section{Comparison to an epidemiological-based contagion model}

As described in detail in Sects. 3 and 4, the ASCRIBE model presented in this paper is an interaction-based model that draws from social contagion theories of emotion and other mental states, such as beliefs and intentions. The final model resembles the dynamics properties as they are found in thermodynamic systems, for example heat diffusion by the interaction of bodies and radiation. A different approach to modelling contagion is used by epidemiological models, which are traditionally well suited to describe phenomena such as the disease spread

See http://www.few.vu.nl/ tbosse/may4/. This URL contains two animations: one in which only the result of the model with contagion is shown, and one in which the results of all four models are shown together. 


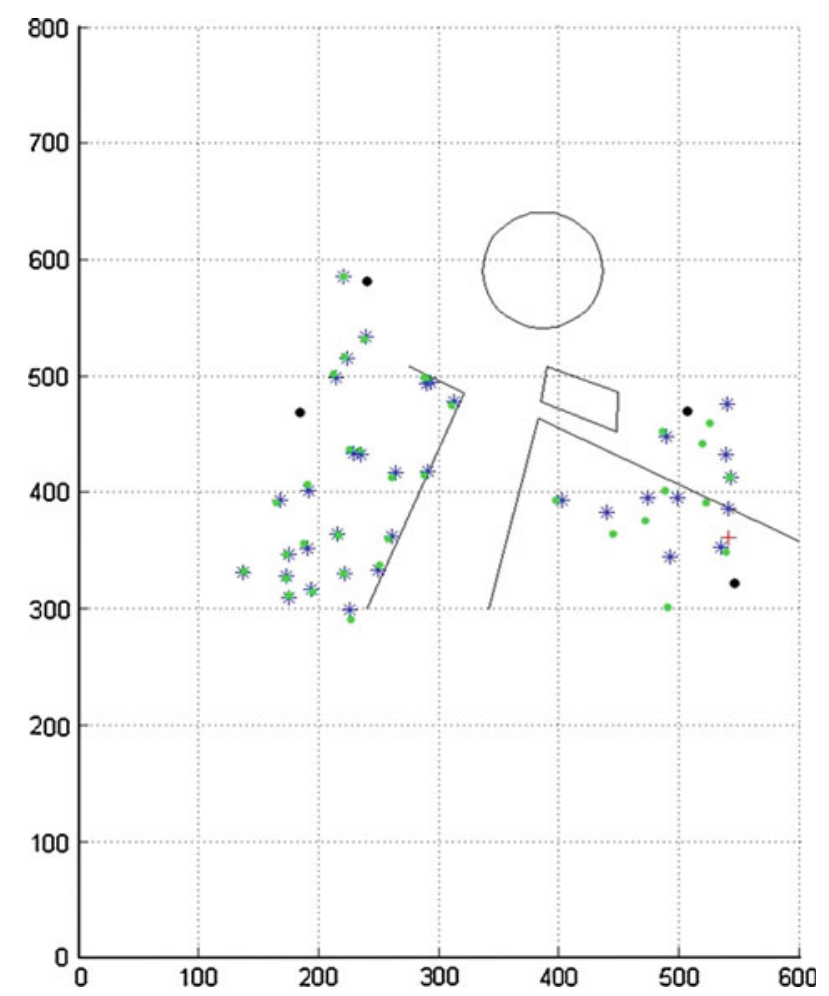

Fig. 11 Screenshot of the simulation. Units displayed on the axes are in pixels, where 5.15 pixels equals $1 \mathrm{~m}$

(e.g., [17]) or innovation diffusion [46], but are also heavily applied to different types of social contagion (e.g., [49]). One example of an epidemiological model that has been applied to social contagion is the Durupinar model [18]. This model uses probabilistic thresholds to determine the likelihood of emotion 'infections' between people in social interactions.

In recent work, Tsai et al. [52] have compared the ASCRIBE and the Durupinar model in an evacuation simulation where both models were tested on their ability to reproduce the dynamics that occurred in an existing crowd panic scene. The simulation was run in ESCAPES [53], which is a multiagent evacuation simulation tool that features different agent types and emotional, informational and behavioural interactions. For this comparison, an earlier and simpler version of ASCRIBE (as presented in [2]), was used. This model does not include the dynamics between emotions, intentions and beliefs, but focuses only on the spread of emotions. Tsai et al. did however expand the simpler version with a proximity effect that is similar to the one used in the extended ASCRIBE model described in this paper (see Sect. 7).

First, a simulation was run that included 100 pedestrians, who experience a fearful event and as a result are trying to find an exit in a large hallway, The results show that the ASCRIBE model was able to produce more realistic dynamics than the Duruprinar model. In the ASCRIBE model, the proximity effect ensured that agents could only be affected by others in their proximity, whereas in the Durupinar model, contagion was able to spread through the entire population immediately. See [52] for a detailed discussion of these results.

Second, the ASCRIBE model was compared to both the Durupinar model and the ESCAPES model, as a baseline comparison. The ESCAPES model uses a basic model of 
Table 5 Average error (in pixels) in the Amsterdam simulation (table adapted from Tsai et al. [52])

\begin{tabular}{|c|c|c|c|c|c|c|c|c|c|}
\hline Overall Near & & Overall & Near & & Overall & Near & & Overall & Near \\
\hline (a) Base models & & & & & & & & & \\
\hline $\begin{array}{lll}\text { None } & 0.375 & 0.699\end{array}$ & ESCAPES & 0.375 & 0.698 & ASCRIBE & 0.362 & 0.663 & Durupinar & 0.383 & 0.758 \\
\hline Model & & & & Overall & & & & & Near \\
\hline \multicolumn{10}{|l|}{ (b) ESCAPES } \\
\hline Base & & & & 0.375 & & & & & 0.698 \\
\hline Decay & & & & 0.379 & & & & & 0.703 \\
\hline No speed & & & & 0.381 & & & & & 0.721 \\
\hline No prox & & & & 0.385 & & & & & 0.721 \\
\hline Variation & & & & Overall & & & & & Near \\
\hline \multicolumn{10}{|l|}{ (c) ASCRIBE model } \\
\hline Base & & & & 0.362 & & & & & 0.663 \\
\hline Decay & & & & 0.363 & & & & & 0.687 \\
\hline No speed & & & & 0.387 & & & & & 0.767 \\
\hline No prox & & & & 0.414 & & & & & 0.797 \\
\hline Model & & & & Overall & & & & & Near \\
\hline \multicolumn{10}{|l|}{ (d) Dunipinar model } \\
\hline Base & & & & 0.383 & & & & & 0.758 \\
\hline No decay & & & & 0.387 & & & & & 0.771 \\
\hline Speed & & & & 0.388 & & & & & 0.784 \\
\hline Prox & & & & 0.380 & & & & & 0.754 \\
\hline
\end{tabular}

emotion contagion wherein agents inherit the highest fear level of neighbouring agents. The simulations were based on two real scenes: the Amsterdam 4 May scene as described in Sect. 6, and recent protests in Greece, where officers fired tear gas in the middle of a small crowd. In both scenarios, the ASCRIBE model performs equal and mostly superior to the other models, outperforming the Durupinar model with $14 \%$ less error per agent per frame in the Amsterdam scenario, and $12 \%$ less error per agent per frame in the Greece scenario. See Table 5 en 6 (adapted from [52]) for the average errors in pixels (compared to the original video's). Each model shows errors for all agents ('overall') and agents near to the catalyzing event ('near'). The models were run in different parameter settings: as given, with implementations of 'decay' turned on/off, with emotional level impacting speed ignored, and with proximity effects turned off. For a discussion on the scenarios, settings and results please refer to [52].

These results show that the underlying mechanisms used in the ASCRIBE contagion model are well suited to model these kind of contagion problems. It seems that in this case the combination of the proximity effect and the mirroring of emotional states yields the most promising results. Future studies will have to show whether the addition of belief and intention dynamics are able to further decrease the error rate.

\section{Discussion}

This paper has presented the computational model ASCRIBE for collective decision making based on neural mechanisms revealed by recent developments in social neuroscience; 
Table 6 Average error (in pixels) in the Greece simulation (table adapted from Tsai et al. [52])

\section{Model}

Error

\begin{tabular}{lc} 
(a) Base models & \\
None & 1.635 \\
ESCAPES & 1.478 \\
ASCRIBE & 1.478 \\
Durupinar & 1.656 \\
(b) ESCAPES & \\
Base & 1.478 \\
Decay & 1.474 \\
No speed & 1.567 \\
No prox & 1.658 \\
\hline Variation & Error \\
\hline
\end{tabular}

(c) ASCRIBE

Base

1.478

Decay

1.466

No speed

1.653

No prox 1.660

Model

Error

(d) Durupinar

Base

1.656

No decay

Speed

1.653

Prox

1.669

1.654

e.g., $[6,7,16,26,33]$. These mechanisms explain how mutual adaptation of individual mental states can be realized by social interaction. They do not only enable intentions to converge to an emerging common decision, but at the same time enable to achieve shared underlying individual beliefs and emotions. Therefore a situation can be achieved in which a common decision that for each individual is considered in agreement with the own beliefs and feelings can be made, thus achieving a solid personal grounding and robustness of the decision. More specifically, this model for collective design making involves on the one hand individual beliefs, emotions and intentions, and on the other hand interaction with others involving mirroring of such mental states; e.g., [33,45,51]. As shown in Fig. 1 and in Table 1, the model involves seven types of interactions: three types of mirroring interactions between different persons, and within each person four types of interactions between the individual mental states. By exploiting knowledge from social neuroscience a biologically plausible, humanlike agent-based computational model was obtained, as was aimed for. Such a model can be used not only for prediction, but also to gain insight in the dynamics of social interaction mechanisms and their emergent properties as described informally in a noncomputational manner in neurological literature.

In earlier work presented in [31] a simpler model for decision making was introduced in which only decision options and emotions associated to them, and their mutual interaction play a role, and no fear, nor interactions with beliefs. This model covers only three of the seven types of interaction of the currently presented model. The overlap is mainly in the somatic marking of intentions for decision options. In [29] a model was introduced in which only emotions and information and their mutual interaction play a role, and no decision-making. The equations for the dynamics of $\delta, \eta$, and $\beta$ were adopted from this paper. 
Moreover, it was discussed how empirical data has been extracted from available video material and witness reports of the May 4 incident in Amsterdam. Qualitative data about escape panics are rare [27]. Based on these data, it is possible to compare models for crowd behaviour with qualitative data of a real panicking event. In this paper, ASCRIBE has been adapted to construct a model for behaviour in a crowd when a panic spiral occurs. Experiments have been performed in which the model was compared to three other models, namely (1) a baseline model where the agents do not move at all, (2) a model by Helbing et al. [27], and (3) a variant of the model where parameters related to contagion were set in such a way that there was no contagion at all; in this case the movement of individuals is only determined by their individual state. In the full ASCRIBE model, mutual influencing took place because emotions, beliefs and intentions were spreading to persons nearby. When comparing the simulations of the four models with the most optimal settings for certain parameters, the variant with contagion had the lowest average error rate per time step ( 0.54 instead of $0.59,0.66$, and 0.87 for Helbing, without contagion, and baseline, respectively). Statistical analysis confirmed the significant differences between the models, in particular for the second part of the scenario. Thus, it is shown that the contagion of mental states is an essential element to model the behaviour of crowds in panic situations.

As discussed in Sect. 9, the added value of the contagion of mental states can be exploited well in the chosen scenario, because of some specific characteristics of this scenario. In particular, the fact that part of the crowd stands still during the first part of the scenario, and only starts to move after they observe (and are probably influenced by) the behaviour of others is a phenomenon that is well suited to be simulated by means of contagion mechanisms. Based upon our analysis, this is the main reason why the ASCRIBE model performs better than the other three models (in which these mechanisms are lacking) in this experiment. Note that this does not necessarily mean that it performs better than, e.g., the Helbing model in other scenarios. A more extensive comparison between these two models for various new scenarios would be an interesting direction for follow-up research.

Previous works have presented several models for crowd behaviour. As mentioned above, an influential paper has been written by Helbing and colleagues [27], in which a mathematical model for crowd behaviour in a panic situation is presented, based on physics theories and socio-psychological literature. This model is based on the principle of particle systems, in which forces and collision preventions between particles are important. This approach is often used for simulating crowd behaviour in virtual environments [48,54]. In [5] the model of [27] is extended by adding individual characteristics to agents, such as the need for help and family membership. In both models, there are no individual emotion, belief and intention states that play a role. In contrast, in [36] an agent has an 'emotional status', which determines whether agents walk together (i.e. it influences group formation). The emotional status of an agent can change when to agents meet. An even further elaborated role of emotional and psychological aspects in a crowd behaviour model can be found in [38]. In this model, several psychological aspects influence the decision making of individual agents, for example, motivation, stress, coping, personality and culture. In none of the models presented above, there is contagion of emotional or other mental states between people. Also, no evaluation with real qualitative data has been performed. One of the most developed tools for crowd simulation, which also incorporates mental states, is ESCAPES [53]. This system, which specifically targets evacuation scenarios, has several similarities with the approach shown here. In Section 10 results of a previous study are shown that compares the ASCRIBE model with the ESCAPE model and the epidemiological-based Durupinar model [52]. These results show that the ASCRIBE model is well equipped to model realistic contagion of emotions. Future 
work will explore the possibilities to incorporate the detailed mechanisms for contagion of mental states presented here into ESCAPES.

Moreover, in the future, further parameter tuning experiments are planned to study the effect of the parameters that were fixed as default values in the current experiments. The aim is to explore whether even more realistic simulations can be achieved by exploiting the details of the model for contagion of emotions, beliefs and intentions in a more differentiated form. This work has, for reasons of simplicity and clarity, focused on homogeneous groups of agents. However, the model accounts for various personality settings. Further research will examine how persons with different personalities can influence the contagion process.

Acknowledgments This research has partly been conducted as part of the FP7 ICT Future Enabling Technologies program of the European Commission under grant agreement No. 231288 (SOCIONICAL). Furthermore, we would like to thank Milind Tambe and Jason Tsai from the University of Southern California for the fruitful discussions.

Open Access This article is distributed under the terms of the Creative Commons Attribution License which permits any use, distribution, and reproduction in any medium, provided the original author(s) and the source are credited.

\section{References}

1. Bechara, A., Damasio, A. (2004). The somatic marker hypothesis: A neural theory of economic decision. Games and Economic Behavior, 52, 336-372.

2. Bosse, T., Duell, R., Memon, Z. A., Treur, J., \& Wal, C. N. V. D. (2009). A multi-agent model for mutual absorption of emotions. In: European council on modeling and simulation, ECMS 2009 (pp. 212-218).

3. Bosse, T., Hoogendoorn, M., Klein, M., Treur, J., \& van der Wal, N. (2011). Agent-based analysis of patterns in crowd behaviour involving contagion of mental states. In: IEA/AIE 2011. Heidelberg: Springer.

4. Bosse, T., Memon, Z. A., Treur, J., \& Umair, M. (2009). An adaptive human-aware software agent supporting attention-demanding tasks. In: J.-J. Yang, M. Yokoo, T. Ito, Z. Jin, \& P. Scerri (Eds.), Proceedings of the 12th international conference on principles of practice in multi-agent systems PRIMA'09, Lecture Notes in AI, vol. 5925 (pp. 292-307). Heidelberg: Springer.

5. Braun, A., Musse, S. R., de Oliveira, L. P. L., \& Bodmann, B. E. J. (2003). Modeling individual behaviors in crowd simulation. In: The 16th international conference on computer animation and social agents CASA 2003 (pp.143-147). New Jersey: IEEE Press.

6. Cacioppo, J. T., \& Berntson, G. G. (2005). Social neuroscience. New York: Psychology Press.

7. Cacioppo, J. T., Visser, P. S., \& Pickett, C. L. (2006). Social neuroscience: People thinking about thinking people. Cambridge: MIT Press.

8. Chen, M., \& Bargh, J. A. (1999). Consequences of automatic evaluation: Immediate behavioral predispositions to approach or avoid the stimulus. Personality \& Social Psychology Bulletin, 25, 215-224.

9. Côté, S. (2005). Reconciling the feelings-as-information and hedonic contingency models of how mood influences systematic information processing. Journal of Applied Social Psychology, 35, 1656-1679.

10. Damasio, A. (1994). Descartes' error: Emotion, reason and the human brain. London: Papermac.

11. Damasio, A. (1996). The somatic marker hypothesis and the possible functions of the prefrontal cortex. Philosophical Transactions of the Royal Society: Biological Sciences, 351, 1413-1420.

12. Damasio, A. (1999). The feeling of what happens. Body and emotion in the making of consciousness. New York: Harcourt Brace.

13. Damasio, A. (2003). Looking for spinoza. London: Vintage Books.

14. Damasio, A., \& Meyer, K. (2008). Behind the looking-glass. Nature, 454, 167-168.

15. Decety, J. \& Cacioppo, J. T. (Eds.). (2010). The handbook of social neuroscience. Oxford: Oxford University Press.

16. Decety, J., \& Ickes, W. (2009). The social neuroscience of empathy. Cambridge: MIT Press.

17. Dodds, P., \& Watts, D. J. (2005). A generalized model of social and biological contagion. Journal of Theoretical Biology, 232(4), 587-604. 
18. Durupinar, F. (2010). From audiences to mobs: Crowd simulation with psychological factors. PhD Dissertation, Bilkent University, Department of Computer Engineering, Ankara.

19. Ekman, P., \& Davidson, R. J. (Eds.). (1994). The nature of emotion: Fundamental questions. New York: Oxford University Press.

20. Ekman, P., \& Friesen, W. V. (1975). Unmasking theface. A guide to recognizing emotions from facial clues. Englewood Cliffs: Prentice-Hall.

21. Frederickson, B. L., \& Branigan, C. (2001). The role of positive emotions in positive psychology: The broaden-and-build theory of positive emotions. American Psychologist, 56, 218-226.

22. Frederickson, B. L., \& Branigan, C. (2005). Positive emotions broaden the scope of attention and thought-action repertoires. Cognition and Emotion, 19, 313-332.

23. Frijda, N. H. (1986). The emotions. Cambridge: Cambridge University Press.

24. Frijda, N. H., Kuipers, P., \& ter Schure, E. (1989). Relations among emotion, appraisal, and emotional action readiness. Journal of Personality and Social Psychology, 57, 212-228.

25. Guten, S., \& Allen, V. L. (1972). Likelihood of escape, likelihood of danger, and panic behavior. Journal of Social Psychology, 87, 29-36.

26. Harmon-Jones, E., \& Winkielman, P. (Eds.). (2007). Social neuroscience: Integrating biological and psychological explanations of social behavior. New York: Guilford.

27. Helbing, D., Farkas, I., \& Vicsek, T. (2000). Simulating dynamical features of escape panic. Nature, 407(6803), 487-490.

28. Helbing, D., \& Molnar, P. (1995). Social force model for pedestrian dynamics. Physical Review E, 51, 4282-4286.

29. Hoogendoorn, M., Treur, J., Wal van der, C. N., \& van Wissen, A. (2010). An agent-based model for the interplay of information and emotion in social diffusion. In: Proceedings of the 10th IEEE/WIC/ACM international conference on intelligent agent technology, IAT'10 (pp. 439-444). New York: IEEE Computer Society Press.

30. Hoogendoorn, M., Treur, J., Wal van der, C. N., \& van Wissen, A. (2010). Modelling the interplay of emotions, beliefs and intentions within collective decision making based on insights from social neuroscience. In: K. K. W. Wong, B. S. U. Mendis \& A. Bouzerdoum (Eds.), Proceedings of the 17th international conference on neural information processing, ICONIP'10. Lecture Notes in Artificial Intelligence, vol. 6443 (pp. 196-206). New York: Springer.

31. Hoogendoorn, M., Treur, J., Wal van der, C. N., \& van Wissen, A. (2010). Modelling the emergence of group decisions based on mirroring and somatic marking. In: Y. Yao, R. Sun, T. Poggio, J. Liu, N. Zhong, \& J. Huang (Eds.), Proceedings of the second international conference on brain informatics, BI'10, LNAI, vol. 6334 (pp. 29-41). Heidelberg: Springer.

32. Iacoboni, M. (2005). Understanding others: Imitation, language, empathy. In S. Hurley \& N. Chater (Eds.), Perspectives on imitation: From cognitive neuroscience to social science (Vol. 1, pp. 77-100). Cambridge: MIT Press.

33. Iacoboni, M. (2008). Mirroring people. New York: Farrar, Straus \& Giroux.

34. Izard, C. E. (1994). Innate and universal facial expressions: Evidence from developmental and cross-cultural research. Psychological Bulletin, 115, 288-299.

35. Kelly, H. H., Condry, J. C., Dahlke, A., \& Hill, A. H. (1965). Collective behavior in a simulated panic situation. Journal of Experimental Social Psychology, 1, 20-54.

36. Musse, S. R., \& Thalmann, D. (1997). A model of human crowd behavior: Group inter-relationship and collision detection analysis. Computer Animation and Simulation, 97, 39-51.

37. Pan, X., Han, C., Dauber, K., \& Law, K. (2006). Human and social behaviour in computational modeling and analysis of egress. Automation in Construction, 15, 448-461.

38. Pelechano, N., O’brien, K., Silverman, B., \& Badler, N. (2005). Crowd simulation incorporating agent psychological models, roles and communication. In: First international workshop on crowd simulation, V-CROWDS'05 (pp. 21-30). Lausanne: V-CROWDS.

39. Pineda, J. A. (Ed.). (2009). Mirror neuron systems: The role of mirroring processes in social cognition. Totowa: Humana Press.

40. Plutchik, R. (1980). Emotion: A psychoevolutionary synthesis. New York: Harper and Row.

41. Quarantelli, E. L. (1954). The nature and conditions of panic. American Journal of Sociology, 60, 267275.

42. Rizzolatti, G. (2005). The mirror-neuron system and imitation. In S. Hurley \& N. Chater (Eds.), Perspectives on imitation: From cognitive neuroscience to social science (Vol. 1, pp. 55-76). Cambridge: MIT Press.

43. Rizzolatti, G., \& Craighero, L. (2004). The mirror-neuron system. Annual Review of Neuroscience, 27, 92-169. 
44. Rizzolatti, G., Fogassi, L., \& Gallese, V. (2001). Neuro-physiological mechanisms underlying the understanding and imitation of action. Nature Review of Neuroscience, 2, 661-670.

45. Rizzolatti, G., \& Sinigaglia, C. (2008). Mirrors in the brain: How our minds share actions and emotions. Cambridge: Oxford University Press.

46. Rogers, E. M. (1962). Diffusion of innovations. New York: The Free Press.

47. Rotteveel, M., \& Phaf, R. H. (2004). Automatic affective evaluation does not automatically predispose for arm flexion and extension. Emotion, 4, 156-172.

48. Sakuma, T., Mukai, T., \& Kuriyama, S. (2005). Psychological model for animating crowded pedestrians. Computer Animation and Virtual Worlds, 16, 343-351.

49. Schelling, T. C. (1973). Hockey helmets, concealed weapons, and daylight saving: a study of binary choices with externalities. Journal of Conflict Resolution, 17, 381-428.

50. Sorenson, H. W. (1980). Parameter estimation: Principles and problems. New York: Marcel Dekker.

51. Striano, T., \& Reid, V. (2009). Social cognition: Development, neuroscience, and autism. Hoboken: Wiley-Blackwell.

52. Tsai, J., Bowring, E., Marsella, S., \& Tambe, M., et al. (2011). Empirical evaluation of computational emotional contagion models. In H. Högni Vilhjálmsson (Ed.), IVA 2011, LNAI 6895. Berlin, Heidelberg: Springer.

53. Tsai, J., Fridman, N., Bowring, E., Brown, M., Epstein, S., Kaminka, G., Marsella, S., Ogden, A., Rika, I., Sheel, A., Taylor, M.E., Wang, X., Zilka, A., \& Tambe, M.: ESCAPES-Evacuation simulation with children, authorities, parents, emotions, and social comparison. In: K., Tumer, P., Yolum, L., Sonenberg, \& P., Stone (Eds.), Proceedings of the 10th international conference on autonomous agents and multiagent systems (AAMAS 2011). Valencia: Innovative Applications Track.

54. Ulicny, B., \& Thalmann, D. (2001). Crowd Simulation for interactive virtual environments and VR training systems. In: Proceedings of the eurographics workshop on animation and simulation'01 (pp. 163-170). Heidelberg: Springer. 